\title{
Nanodiamonds as novel nanomaterials for biomedical applications: drug delivery and imaging systems
}

This article was published in the following Dove Press journal:

International Journal of Nanomedicine

8 January 2013

Number of times this article has been viewed

\section{Randeep Kaur \\ Ildiko Badea}

Drug Design and Discovery Research Group, College of Pharmacy and Nutrition, University of Saskatchewan, Saskatoon, Saskatchewan, Canada
Correspondence: Ildiko Badea College of Pharmacy and Nutrition, University of Saskatchewan, I I0 Science Place, Thorvaldson Building, Room G22, Saskatoon, Saskatchewan, S7N 5C9, Canada

Tel +I 3069666349

Fax + I 3069666377

Email ildiko.badea@usask.ca

\begin{abstract}
Detonation nanodiamonds (NDs) are emerging as delivery vehicles for small chemical drugs and macromolecular biotechnology products due to their primary particle size of 4 to $5 \mathrm{~nm}$, stable inert core, reactive surface, and ability to form hydrogels. Nanoprobe technology capitalizes on the intrinsic fluorescence, high refractive index, and unique Raman signal of the NDs, rendering them attractive for in vitro and in vivo imaging applications. This review provides a brief introduction of the various types of NDs and describes the development of procedures that have led to stable single-digit-sized ND dispersions, a crucial feature for drug delivery systems and nanoprobes. Various approaches used for functionalizing the surface of NDs are highlighted, along with a discussion of their biocompatibility status. The utilization of NDs to provide sustained release and improve the dispersion of hydrophobic molecules, of which chemotherapeutic drugs are the most investigated, is described. The prospects of improving the intracellular delivery of nucleic acids by using NDs as a platform are exemplified. The photoluminescent and optical scattering properties of NDs, together with their applications in cellular labeling, are also reviewed. Considering the progress that has been made in understanding the properties of NDs, they can be envisioned as highly efficient drug delivery and imaging biomaterials for use in animals and humans.
\end{abstract}

Keywords: dispersion, surface functionalization, toxicity, carriers, fluorescence, light scattering

\section{Introduction}

Elemental carbon has six electrons with an electronic configuration of $1 s^{2} 2 s^{2} 2 p^{2}$ in its ground state. The two electrons of the 1 s orbital are core electrons, while the other four are valence electrons. $\mathrm{Sp}^{2}$ hybridization of carbon atoms leads to the formation of the two-dimensional planar hexagonal structure of graphite. In this type of arrangement, the central carbon is linked to three other carbon atoms by $\sigma$-bonds, with the remaining electron of the $\mathrm{p}_{\mathrm{z}}$ orbital forming a delocalized cloud of $\pi$ electrons over the graphitic structure. $\mathrm{Sp}^{3}$ hybridization of carbon atoms forms a rigid diamond structure with tetrahedral symmetry, where all the four valence electrons of the carbon atom each form a $\sigma$ bond with the neighboring carbon atoms. The general lack of free electrons in the bulk structure of diamond accounts for their inertness. However, the surface structure of the material is different from the bulk, due to the relaxation at the surface and the need to terminate the bonds. Thus, the terminating structure on diamond surfaces involves univalent species such as $\mathrm{H}$ or $\mathrm{OH}$ (hydrogen or hydroxyl) or any deliberate terminating groups for both nano and larger gem diamonds (synthetic and natural).

Nanodiamonds (NDs) have attracted a great deal of scientific and technological interest due to their unique structural, chemical, biological, mechanical, and 
optical properties. Based upon their primary particle sizes, Shenderova and McGuire classified NDs into nanocrystalline particles ( 1 to $\geq 150 \mathrm{~nm}$ ), ultrananocrystalline particles ( 2 to $10 \mathrm{~nm}$ ), and diamondoids ( 1 to $2 \mathrm{~nm}$ ). ${ }^{1}$ Ultrananocrystalline diamond particles are of particular interest for biomedical applications and focus has been largely placed on detonation NDs with a primary particle size of 4 to $5 \mathrm{~nm}$. $^{2}$ Figure 1 shows the classification of diamonds based on synthetic production methods. The types of diamonds include chemical vapor deposition diamonds, high-pressure high-temperature diamonds, and detonation NDs.

Deposition of carbon vapors on a diamond or non-diamond substrate leads to the formation of diamond films; ${ }^{3}$ the nanocrystalline and ultrananocrystalline types of these are of major interest, due to their superior mechanical and wear resistant properties. Depending on the growth precursors (hydrogen-rich and carbon-poor precursors) and other deposition parameters such as biasing voltage, surface temperature, and film pressure, the grain size of nanocrystalline diamond films can vary from 5 to $100 \mathrm{~nm} \cdot{ }^{4,5}$ Ultrananocrystalline diamond films with a much smaller grain size of 3 to $5 \mathrm{~nm}$ are usually synthesized under hydrogen-poor and argon-rich conditions..$^{6-8}$ In addition to the difference in grain size, these two types of diamond films also differ in their $\mathrm{sp}^{2}$ carbon content. Nanocrystalline diamond films grown in a methanerich environment possess a $\mathrm{sp}^{2}$-bonded carbon structure of up to $50 \%$, which decreases drastically to less than $0.1 \%$ with decrease in the amount of methane. ${ }^{9}$ For the ultrananocrystalline diamond films, the $\mathrm{sp}^{2}$-bonded carbon fraction ranges from $2 \%$ to $5 \%$. ${ }^{9}$ Scanning transmission electron microscopic images of nanocrystalline ${ }^{4}$ and ultrananocrystalline ${ }^{10}$ diamond films are shown in Figure 2.

For coating biomedical implants, ultrananocrystalline diamond films are emerging as substrates superior to those of silicon, platinum, and quartz. ${ }^{11,12}$ This advantage is due to their low cytotoxicity, dense structure, high surface roughness, $\mathrm{sp}^{2}$ structural boundaries and surface dangling bonds. ${ }^{11,12}$ These films also exhibit the desired characteristics for producing micro-electro-mechanical devices due to their robustness, high Young's modulus, high acoustic velocity, high resistance to fracture, and low friction coefficient $(\sim 0.01$ to 0.1$) .{ }^{13-16}$ Similarly, the high stability, superior electrochemical properties,

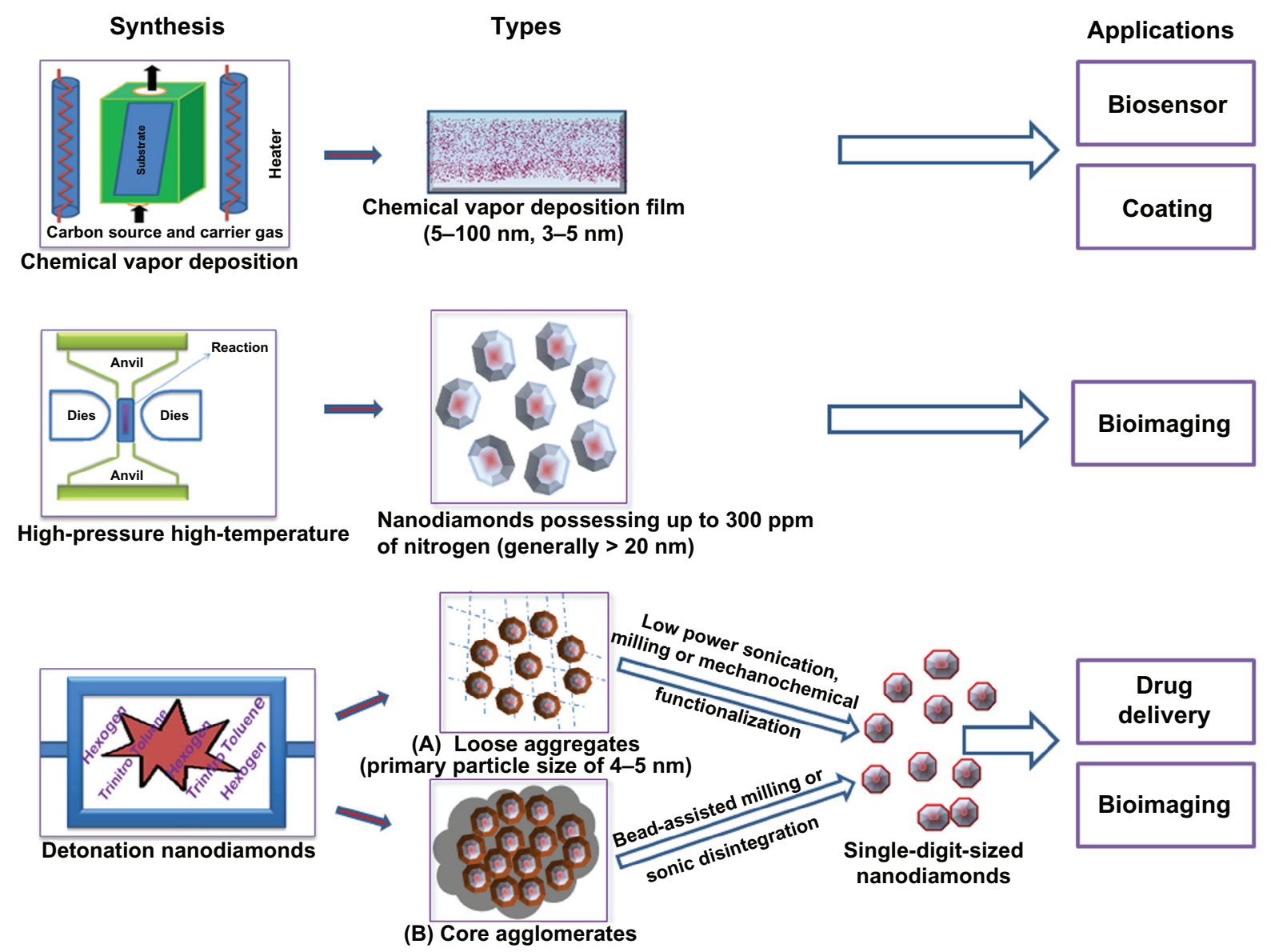

Figure I A schematic representation of nanodiamond types based on their methods of synthesis and applications. 

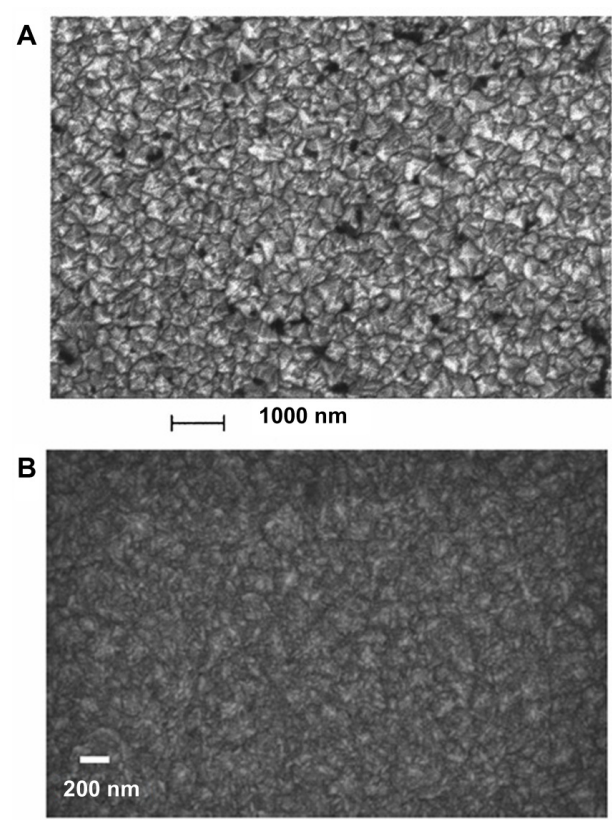

Figure 2 Scanning electron microscopic image of (A) nanocrystalline ${ }^{4}$ and (B) ultrananocrystalline ${ }^{10}$ diamond films grown on silicon substrate.

A is reprinted with permission from: Philip J, Hess P, Feygelson T, et al. Elastic, mechanical, and thermal properties of nanocrystalline diamond films. J Appl Phys. 2003;93(4):2164-217I,4 Copyright (2003), American Institute of Physics. B is reprinted with permission from: Sumant AV, Grierson DS, Gerbi JE, Carlisle JA, Auciello O, Carpick RW. Surface chemistry and bonding configuration of ultrananocrystalline diamond surfaces and their effects on nanotribological properties. Phys Rev B Condens Matter Mater Phys. 2007;76(23):235429-I-235429$11,{ }^{10}$ Copyright (2007) by the American Physical Society.

selectivity in binding biological materials, and biocompatibility of the nanocrystalline diamond films have opened up their potential in the development of biosensors. ${ }^{17,18}$

Synthetic diamonds produced by a high-pressure hightemperature technique could contain up to 100 to $300 \mathrm{ppm}$ of nitrogen. ${ }^{19-22}$ High-energy irradiation of these diamond particles causes damage by knocking a diamond carbon atom out of the structure, creating vacancies $(\mathrm{V}) .{ }^{23}$ Upon thermal annealing, these vacancies move closer to the nitrogen (N) centers to form nitrogen-vacancy (N-V) color defect centers. ${ }^{23}$ These centers are responsible for the emission of fluorescence. ${ }^{24}$ Hence, high-pressure high-temperature synthetic diamonds have been widely explored as imaging agents in cellular models..$^{19,22,25}$ However, the high production cost and the general inability to produce diamond particles smaller than $20 \mathrm{~nm}$ are significant barriers to their use in biomedical sciences.

The third category of diamonds, detonation NDs, also known as "ultradispersed diamonds," are synthesized relatively inexpensively on a large scale by the detonation of carbon explosives with a $\mathrm{C}_{\mathrm{m}} \mathrm{H}_{\mathrm{n}} \mathrm{N}_{\mathrm{o}} \mathrm{O}_{\mathrm{p}}$ composition. ${ }^{26}$ The explosive mixtures are required to have an overall negative oxygen balance in the detonation chamber ${ }^{26}$ to prevent the complete combustion of the carbon into its gaseous products. Generally, a mixture of trinitrotoluene and hexogen in a mass ratio ranging from 40:60 to $70: 30$ is utilized. ${ }^{27} \mathrm{~A}$ shock wave produced by the detonator compresses and heats the composition mixture, leading to its explosion. ${ }^{26} \mathrm{~A}$ large amount of energy is released during this exothermic process, which consequently raises the temperature in the detonation chamber to $3000^{\circ} \mathrm{C}-4000^{\circ} \mathrm{C}$ and the pressure to $20-30 \mathrm{GPa}$, to favor the synthesis of the diamond particles (Figure 3). ${ }^{28,29}$ After the detonation is complete, a rapid cooling is achieved, either by using an inert cooling gas (dry synthesis) or water (wet synthesis). ${ }^{29}$ The cooling of the detonation products is crucial to minimize transformation of the diamond phase into graphitic forms, in accordance with the phase diagram (Figure 3). ${ }^{28,29}$ The average primary particle size of NDs generated by this technique is $\sim 4$ to $5 \mathrm{~nm}$ (Figure 4). ${ }^{28,30}$

Although the primary particle size of NDs obtained by the detonation technique is well suited for biomedical studies, the detonation products need to be extensively purified. Depending on the materials and matrices present during their production, detonation NDs can contain oxides and carbides, including those of iron, chromium, silicon, calcium, copper, potassium, titanium, and sulfur, in addition to carbon soot. ${ }^{26}$ To remove surface metallic impurities, NDs are treated with classic acidic treatments consisting of sulfuric acid and its mixtures with nitric acid or potassium dichromate. ${ }^{31}$

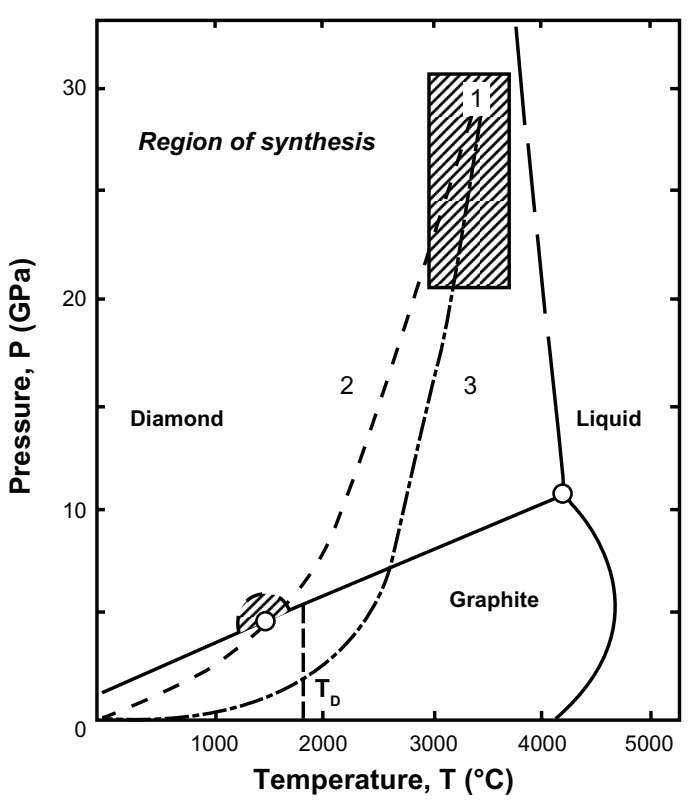

Figure 3 Phase diagram for carbon depicting the pressure and temperature requirements for synthesis of detonation nanodiamond (1); cooling profile of products produced by wet detonation synthesis (2) and dry detonation synthesis (3). ${ }^{29}$ Notes: " $T_{D}$ " represents Debye temperature.

Reproduced courtesy of IOP Publishing Ltd, from: Baidakova M, Vul A. New prospects and frontiers of nanodiamond clusters. J Phys D Appl Phys. 2007;40(20):6300-63II. 


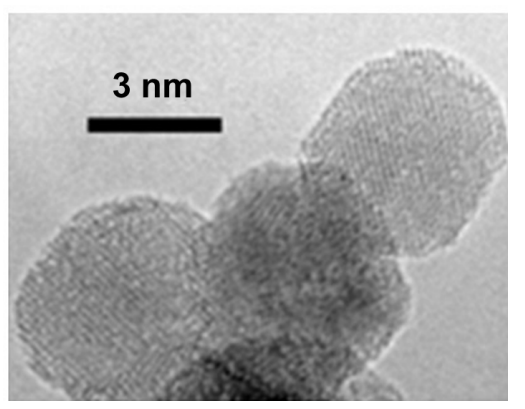

Figure 4 High-resolution transmission electron microscopic image of detonation nanodiamonds.

Note: Image is courtesy of Bogdan Palosz, IHPP, Warsaw, Poland.

A hydrofluoric acid and nitric acid combination has also been used to eliminate metallic contaminants from the particles. ${ }^{32}$ The oxidization and subsequent removal of $\mathrm{sp}^{2}$-bonded carbon structures, present either in amorphous or graphitic forms, is achieved by the use of liquid oxidizers such as sodium peroxide, a chromium trioxide and sulfuric acid mixture, or a nitric acid and hydrogen peroxide mixture. ${ }^{31}$ Thermal oxidation process uses temperatures of $400^{\circ} \mathrm{C}-430^{\circ} \mathrm{C}$ to allow the oxidation of $\mathrm{sp}^{2}$-bonded carbon species in the air, with negligible alteration in $\mathrm{sp}^{3}$-bonded carbon structures. ${ }^{33}$ These temperature requirements for the selective oxidation of $\mathrm{sp}^{2}-$ bonded carbon species were confirmed by Pichot et al. ${ }^{32}$ The oxidation of NDs at a high temperature using air containing ozone is another approach that results in the removal of the majority of $\mathrm{sp}^{2}$-hybridized carbon structures. ${ }^{34}$ The ozone-air treatment, also known as the "gas phase treatment," is ecological and efficient, as the purification of NDs is achieved without using the corrosive liquid oxidizers. ${ }^{34}$

Although the size, shape and surface properties of NDs are determined by the nature of explosion and purification conditions, their basic structure follows a core and shell model. ${ }^{2}$ The diamond carbon forms the inert core and the surface shell is partially comprised of graphitic structures. ${ }^{30,35,36}$ In addition, a wide variety of functional groups such as carboxyl, hydroxyl, lactone, anhydride, ketone, and ether can be present on the surface of these ND particles. ${ }^{36,37}$ X-ray diffraction is one of the most utilized techniques for characterizing NDs, including in terms of their size, structure, and composition. ${ }^{38-40}$ Upon annealing the NDs at $1500^{\circ} \mathrm{C}$ for 10 minutes in a vacuum, the diamond $\mathrm{X}$-ray diffraction peak disappears with the emergence of $\mathrm{sp}^{2}$ carbon structure peak. ${ }^{41}$ The crystal lattice parameters and hence the quality of NDs can vary depending upon the synthesis conditions, as determined by $\mathrm{X}$-ray diffraction studies. ${ }^{38}$

The economical large-scale production of detonation NDs provided considerable impetus to technological applications.
For example, the antifriction properties of NDs and their soot make them ideal candidates as wear-protective additives. ${ }^{42}$ The stability of these particles at extreme temperatures had led to applications in composite manufacturing. ${ }^{43}$ The large surface area of NDs is suitable for adsorbing biomolecules, presenting them as an attractive material for isolating proteins ${ }^{44,45}$ and pathogenic microorganisms ${ }^{46}$ However, it was not until the identification and characterization of single-digitsized NDs in the dispersion medium that NDs were applied in biomedical sciences.

\section{NDs in the biomedical field}

The application of NDs in biological sciences requires their stable dispersion in a formulation medium, ability to conjugate with biomolecules, inherent biocompatibility, and ability to penetrate target tissues and cells.

\section{Dispersion of NDs}

Detonation NDs possess a strong propensity to aggregation when dispersed in a liquid formulation medium, hindering their biomedical applications. Several attempts to explain the aggregation phenomenon have focused on surface forces. The harsh conditions in the detonation chamber could create dangling bonds on the ND surfaces. ${ }^{47}$ These free electron surfaces are sufficiently reactive to form functional groups, which then interact via various intermolecular surface forces such as van der Waals and dipole-dipole interactions, and hydrogen bonding. ${ }^{47}$ In addition, these surface groups can also react to create covalent bonds between the primary particles, ${ }^{48}$ producing core aggregates. Another study suggests that electrostatic forces induced by the oppositely charged facets of polyhedral shaped NDs are the primary reason for the creation of aggregates. ${ }^{49}$ This finding was supported later with the aid of electron microscopy and computer simulations. ${ }^{50}$ Other than the surface forces, the "graphitic soot-like structures" around the particles can also bind individual ND particles together into core aggregates (Figure 1). ${ }^{35,36}$

Conventional techniques of disintegration, such as milling, emulsification, and low power sonication, have been ineffective in breaking down the tightly bound core aggregates of NDs to less than $10 \mathrm{~nm} .{ }^{35}$ Therefore, in an attempt to achieve primary-sized NDs, milling was carried out using an excessive amount of $100 \mu \mathrm{m}$ silica beads. ${ }^{35}$ Single-digit-sized NDs were obtained after an hour of sonication of the diluted milled suspension and the stability of the resulting aqueous colloid was maintained for a year. ${ }^{35}$ However, reconstitution with water after drying the nanodispersion resulted in 
reaggregation into aggregates of $2.3 \mu \mathrm{m} .{ }^{35}$ Bead-assisted sonic disintegration using zirconia beads has also gained interest for breaking the ND aggregates in aqueous medium to single-digit-sized particles. ${ }^{51}$ This mechanical technique involves the combination of shear forces induced by beads with their acceleration during cavitation created by intense ultrasonic waves. These primary-sized NDs exhibited very good colloidal stability in polar solvents such as water and dimethyl sulfoxide (DMSO) ${ }^{51}$ However, contamination from the beads and sonotrode material itself can be a barrier for these technologies, ${ }^{35,52}$ in addition to the structural alterations. ${ }^{53}$

Bead-assisted milling ${ }^{51,54}$ or sonication ${ }^{52}$ yielded clear, but dark brown nanodispersions of diamond particles. It should be noted that sonication creates micro bubbles in liquid media, which implode violently, generating a local temperature of $\sim 1900 \mathrm{~K}$ at the bubble-liquid interface. ${ }^{55}$ The shock waves produced during high-power sonication cause high-speed collision of the beads with ND particles, which can lead to the conversion of the diamond carbon into its graphitic form, according to the diamond-graphitic phase transition phenomenon occurring at temperatures greater than 1200 K. ${ }^{56}$ Similarly, a temperature of $1800 \mathrm{~K}$ can be attained during milling collisions when the speed of the blades is between 0.1 and $10 \mathrm{~cm} / \mathrm{s} .{ }^{54}$ This could explain the black coloration of the colloids observed after high-speed milling or high-power bead-assisted probe sonication.

Mechanochemical treatment, which involves the breaking of ND aggregates by mechanical energy and the concurrent loading of electrolytes or surfactants onto a newly created surface, was also explored to achieve stable dispersions with a noticeable size reduction. ${ }^{57}$ However, a primary dispersed particle size of less than $10 \mathrm{~nm}$ was not achieved. ${ }^{57}$ Ultracentrifugation, a contamination-free procedure, resulted in NDs of $4 \mathrm{~nm}$ that formed a stable colloidal dispersion in water. ${ }^{58}$ The yield of single-digit-sized particles was only $1 \%$ after centrifugation of the ND suspension at an acceleration of $2.80 \times 10^{5} \mathrm{~g}$ for 40 minutes. ${ }^{58}$ Increasing the centrifugation time to 240 hours at an acceleration of $2.0 \times 10^{4} \mathrm{~g}$ did not improve the yield significantly, generating $6 \%$ of NDs with an average size of $8 \mathrm{~nm} .{ }^{58}$ Hence, there is still a need for the development of easy and feasible contamination-free techniques that can result in disaggregation of NDs at the laboratory scale.

\section{Surface modification of NDs}

Together, the nano size, larger surface area, and potential for purification with oxidizing agents make NDs a viable candidate for surface functionalization. Figure 5 summarizes approaches used in the past to introduce various functionalities on the surface of NDs. ${ }^{48,59-66}$ One of the earliest modifications of the ND surface involved the generation of surface radicals, which then acted as substrates for synthesizing carboxylic acid $-{ }^{59}$ and dicarboxylic acid- ${ }^{60}$ functionalized NDs. For a high surface loading, it is essential to achieve surface uniformity. Reactions with hydrogen, ${ }^{59,60}$ chlorine, ${ }^{61}$ and fluorine ${ }^{62}$ have been explored to attain surface homogeneity and reactivity enhancement. The surfaces of chlorinated NDs were further modified with hydroxyl, amine, and carbon fluoride groups. ${ }^{61}$ Finally, reactions of NDs with alkyllithium, ethylenediamine, and glycine ethyl ester hydrochloride generated alkyl-, amino-, and glycinesubstituted NDs, respectively. ${ }^{62}$

Surface functionalization has also emerged as a novel treatment for reducing aggregate sizes of NDs. Functionalization with long alkyl chains reduced the particle size from $15 \mu \mathrm{m}$ to $150-450 \mathrm{~nm} .{ }^{63}$ The long alkyl chain-modified NDs showed an enhanced dispersibility in organic solvents compared with their pristine form. ${ }^{63}$ Similarly, surface modification with fluorine contributed to size reduction from 1930 to $160 \mathrm{~nm} .^{62}$ A noticeable size reduction of the micrometer-sized pristine NDs to $\sim 50 \mathrm{~nm}$ was also achieved upon reduction of the ND surface groups with borane. ${ }^{65}$ After being grafted with alkyl silane, these surface-modified NDs were used to synthesize biotinylated NDs with a surface loading of $1.45 \mathrm{mmol} / \mathrm{g} .{ }^{65}$ Lysine molecules attached to the surface of NDs reduced the aggregate size from 1281 to $21 \mathrm{~nm} .{ }^{66}$ The resulting lysinefunctionalized NDs with a surface loading of $1.7 \mathrm{mmol} / \mathrm{g}$ showed a considerably better dispersibility in water compared with carboxylated NDs. ${ }^{66}$

\section{Biocompatibility studies of NDs}

The intrinsic biocompatibility of detonation NDs has been supported by the results of a number of studies, ${ }^{67-72}$ however, some studies have refuted this claim by arguing that NDs can induce toxic responses under certain conditions. ${ }^{73,74}$

The biocompatibility of acid- or base-purified NDs of 2 to $10 \mathrm{~nm}$ was first demonstrated in neuroblastoma, macrophage, keratinocyte, and PC-12 cells. ${ }^{67}$ By considering the mitochondrial activity as a key determinant of cellular viability, NDs up to a concentration of $100 \mu \mathrm{g} / \mathrm{mL}$ were reported not to be cytotoxic over a 24-hour incubation period. ${ }^{67}$ In addition, NDs showed higher biocompatibility than carbon black, and single- and multi-walled carbon nanotubes. ${ }^{67,68}$ When incubated for 24 hours with NDs at the concentration range of 25 to $100 \mu \mathrm{g} / \mathrm{mL}$, no significant cell death occurred in neuro- 


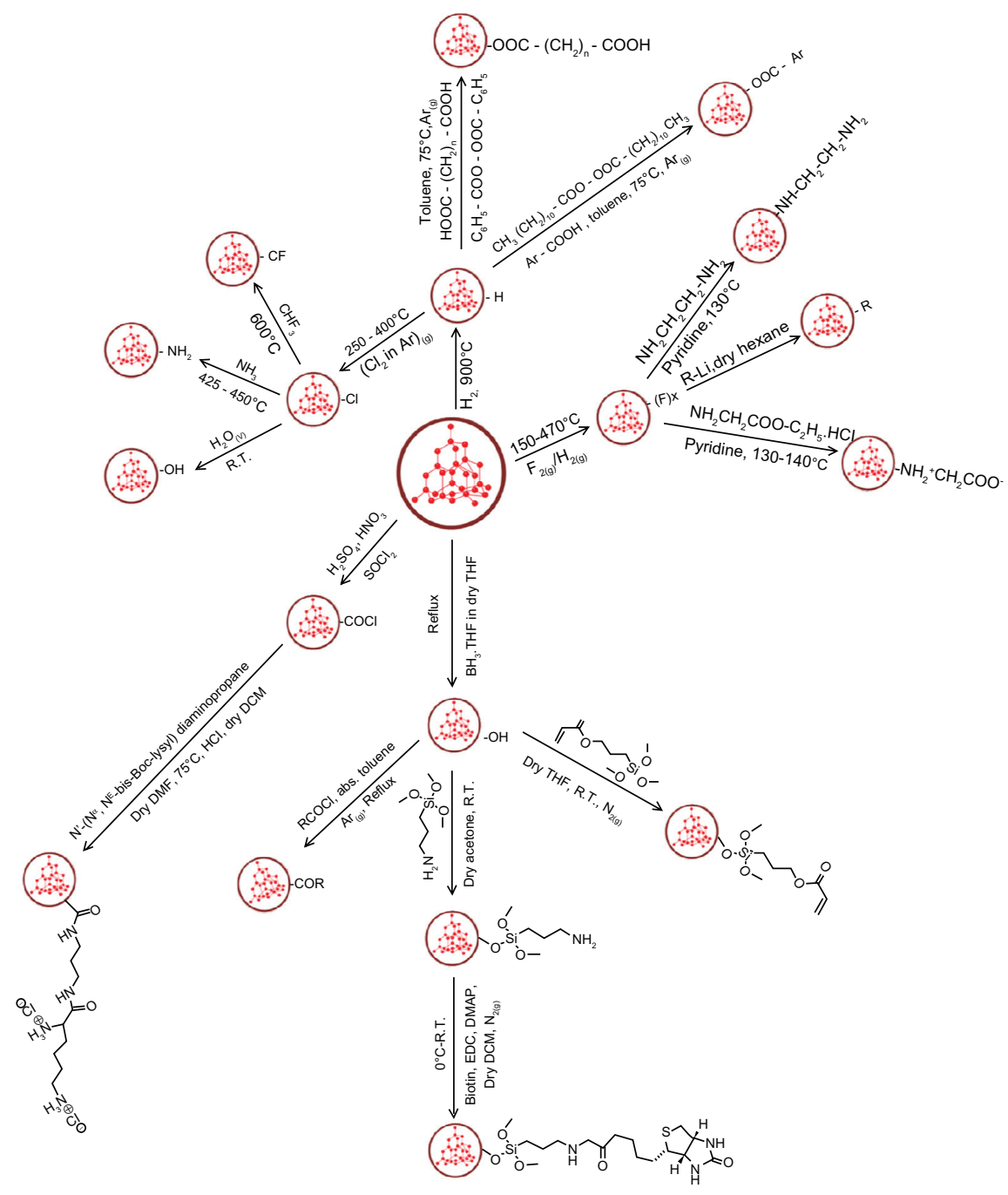

Figure 5 A schematic representation of approaches used for modification of nanodiamond surfaces.

Abbreviations: R.T., room temperature; R, alkyl group; Ar, aromatic group; THF, tetrahydrofuran; DMAP, (dimethylamino)pyridine; EDC, (I-ethyl-3-[(3-dimethylamino) propyl]carbodiimidehydrochloride).

blastoma cells. ${ }^{68}$ Similarly, alveolar macrophages maintained the cellular viability upon exposure for 24 hours to NDs at a concentration of $25 \mu \mathrm{g} / \mathrm{mL} .{ }^{68}$ In addition to mitochondrial function, the inflammatory activity of cells has also been a key determinant of the toxic effects of nanomaterials. ${ }^{72}$ There was no significant increase in the gene expression of inflammation biomarkers, such as interleukin-6, tumor necrosis factor-alpha and nitric oxide synthase, in murine macrophages after incubation with a $100 \mu \mathrm{g} / \mathrm{mL}$ concentration of NDs. ${ }^{72}$ In addition, no significant attenuation in the gene expression level of Bcl-x, which generally diminishes with an increased risk of apoptosis, endorsed the intrinsic biocompatibility of these diamond nanoparticles. ${ }^{72}$

Various studies have demonstrated that the toxicity of NDs can vary depending upon their surface chemistry, ${ }^{74,75}$ the type of cell line, ${ }^{74}$ and the composition of the treatment medium. ${ }^{73}$
The amine-terminated NDs exhibited a higher toxicity ( $22 \%$ cell death) than hydroxyl-terminated NDs $(\sim 11 \%)$ and carboxyl group-terminated NDs $(\sim 7 \%)$ in human embryonic kidney cells at a concentration of up to $200 \mu \mathrm{g} / \mathrm{mL} .^{75}$ The carboxylated NDs did not show any significant genotoxic effects in mouse embryonic fibroblasts ${ }^{76}$ but induced DNA damage in mouse embryonic stem cells, ${ }^{74}$ although to a lesser extent than what occurred with multi-walled carbon nanotubes. ${ }^{77}$ In another instance, a 100\% viability of human cervical cancer (HeLa) cells was observed after incubating them with $0.1-100 \mu \mathrm{g} / \mathrm{mL}$ NDs in a medium containing serum for 24 hours. ${ }^{73}$ In the absence of serum, this viability dropped dramatically to almost $0 \%$ within 6 hours of dosage with $50 \mu \mathrm{g} / \mathrm{mL}$ NDs. ${ }^{73}$ Similar behavior has been also observed with other nanoparticles such as carbon black ${ }^{78}$ and silica. ${ }^{79}$ Although there is no clear explanation of the 
serum-dependent cytotoxicity of nanoparticles so far, several hypotheses have been put forth. It is believed that serum can have a protective effect on the cells by coating the surface of nanoparticles that are thought to have inherent cytotoxic effects. ${ }^{73,78,80}$ While, on one hand, serum is found to enhance the dispersion stability of the nanoparticles, ${ }^{73,78,81}$ on the other, serum promotes aggregation. ${ }^{79}$ The cellular internalization of nanoparticles was observed to be higher in the absence of serum than in its presence, ${ }^{78,79}$ which might be one of the reasons for the lower cellular toxicity of nanoparticles when incubated with serum. Another study found that nanoparticles could adsorb the essential micronutrients from the medium, hence indirectly produce cytotoxic effects by depleting the cellular structures of essential nutrients. ${ }^{82}$ Finally, the serum proteins themselves are essential for the growth of the cell, thus their absence can have a negative effect on overall cell proliferation. However, it is important to note that NDs did not show significant toxicity in some studies, even when incubated with cells in medium lacking serum. ${ }^{67,68}$

In addition to the cellular studies, some in vivo studies have been conducted to evaluate the toxicity of NDs. The intravenous administration of $125 \mathrm{mg}$ of modified NDs did not cause any deaths in rabbits. ${ }^{83}$ Neither the red blood cell count nor the hemoglobin level of the rabbits decreased after 15 minutes of administering $50 \mathrm{mg}$ of modified NDs intravenously. ${ }^{83}$ However, 48 hours after the injection, the levels of biochemical molecules such as total bilirubin, triglyceride, low-density lipoprotein, aspartate aminotransferase, and $\gamma$-glutamyltranspeptidase altered significantly. ${ }^{83}$ In another study, an oral administration of 0.002 to $0.05 \mathrm{wt} \%$ of $\mathrm{ND}$ hydrosols to mice instead of water for 6 months showed no significant abnormalities in growth or organs weight. ${ }^{43}$ The NDs exhibited low pulmonary toxicity and were cleared from the lungs after 90 days of intratracheal instillation in imprinting control region (ICR)-strain mice. ${ }^{84}$ Furthermore, the clearance of XenoFluor ${ }^{\mathrm{TM}}$ 750-labeled NDs from all the body organs of wild-type FVB/N (mouse strain) mice occurred in 3 to 10 days after tail vein injection at a dose of $120 \mu \mathrm{g} .{ }^{85}$

In summary, NDs have shown biocompatibility in various cell lines and some animal models with minimal or no cytotoxicity, demonstrating their potential for biomedical applications. However, the purity, surface chemistry, and dimensions of particles should be considered carefully. More comprehensive and long-term animal studies need to be conducted to verify the safety of NDs before proceeding to human clinical trials.

\section{Cellular uptake of NDs}

A number of studies have demonstrated the ability of NDs to internalize into cells. ${ }^{19,25,67,69-71,86-88}$ Faklaris et al demonstrated that NDs having an average size of $46 \mathrm{~nm}$ internalize into HeLa cells predominantly by clathrin-mediated endocytosis after 2 hours of incubation. ${ }^{88}$ In addition to the clathrinmediated endocytic pathways, NDs of $100 \mathrm{~nm}$ were found to be internalized by cancer and stem cells after 4 hours of incubation via macropinocytosis. ${ }^{76}$ The intracellular localization of the NDs suggests that these nanoparticles have the potential to be employed for the intracellular delivery of therapeutic molecules. While the NDs used in these studies showed only perinuclear localization with no translocation to the nucleus, ${ }^{76,88}$ fenton-treated NDs were detected in the nucleus of HeLa cells. ${ }^{89}$ The internalization of NDs into cells could be dependent upon their surface characteristics, ${ }^{89}$ incubation time, and other physiochemical parameters such as size, shape, and aggregation. Nuclear internalization of NDs opens up the possibility of their use as delivery agents for nucleic acids. Figure 6 summarizes the prominent applications of NDs in the biomedical field.

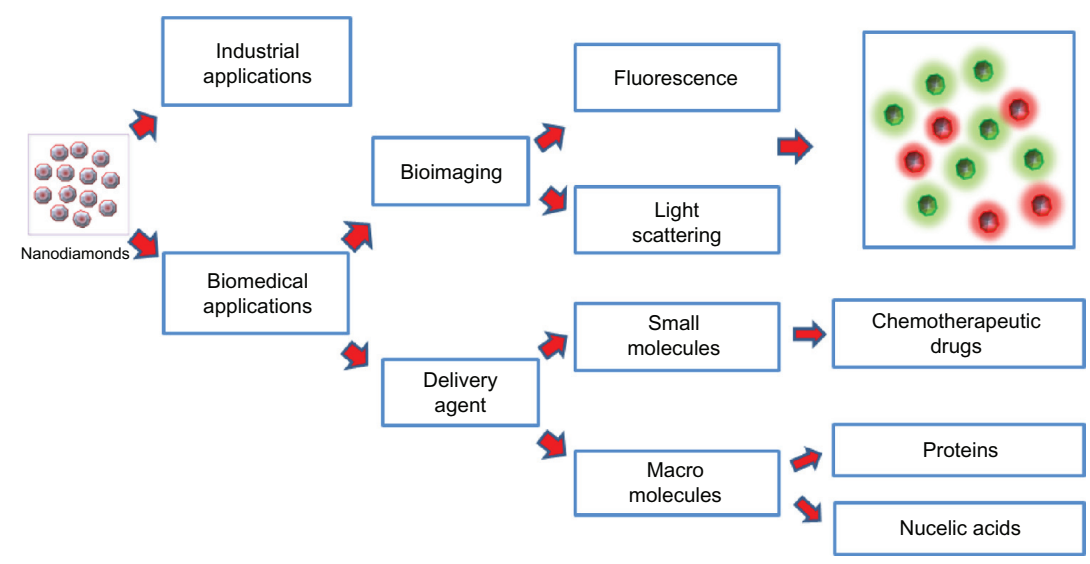

Figure 6 A schematic representation of the applications of the nanodiamonds. 


\section{Applications of NDs as drug delivery agents}

The large surface area of biocompatible NDs, which have several surface functionalities, is ideal for conjugating various biochemical entities. The suitability of the carrier can be projected from its loading capacity as well as from its ability to protect and retain the inherent therapeutic effects of the attached entities. A high loading capacity allows a high concentration of payload to be delivered while using less of the delivery agent itself. In parallel to the loading capacity, the release of payload from the carrier is also important. The feasibility of being able to tune the release of cargo from the carrier is advantageous in developing novel applications of these delivery agents, such as controlled- and sustained-release delivery. Diamond nanoparticles are being investigated globally for improving the intracellular delivery of small molecules and biotechnological products, with a major focus on chemotherapeutic agents. Although some studies have made use of the ND surfaces to bind drugs via chemical bonding, ${ }^{90,91}$ the majority have focused on physical adsorption procedures. ${ }^{72,73,85,92}$ This simple process of loading cargo onto the carrier via physical interactions avoids the use of complex chemical reactions, which, in addition to being high cost, can also affect the therapeutic activity of the attached entity by causing structural alterations. ${ }^{93}$ The use of NDs as a small-molecule delivery agent, using chemotherapeutic drugs as model drugs, has been exemplified by various studies.

\section{Delivery of small molecules}

In 2007, the suitability of NDs to act as a delivery agent of doxorubicin hydrochloride (DOX) was evaluated. ${ }^{72}$ The investigation was based on the rationale that the surface carboxylic and hydroxylic groups of detonation NDs can interact efficiently with the amine groups of DOX via ionic forces when dispersed in aqueous medium. ${ }^{72}$ The surface loading of DOX on NDs increased from 0.5 to $10 \mathrm{wt} \%$ upon addition of $1 \%$ sodium chloride solution to their aqueous dispersion, and the removal of salt favored the release of DOX. ${ }^{72}$ NDs loaded with DOX were suggested to assemble in the form of loose clusters, such that a certain amount of DOX adsorbed on the NDs surface resides within the cavity of the cluster (Figure 7A). ${ }^{72}$ This strategy of drug entrapment in loose aggregates of NDs could provide an advantage by minimizing the systemic adverse effects of naked DOX. ${ }^{72}$ Thus, ND-based delivery systems could overcome the limitation to the use of high concentrations of chemotherapeutic drugs ${ }^{94}$ in cancer treatments. In addition, the lower cytotoxicity of the ND-DOX composite in mouse macrophages and human colorectal cancer cells compared with bare DOX in a 48-hour period could be beneficial in sustained drug release. ${ }^{72}$

Another study, published in 2010, proposed NDs as efficient multifunctional delivery agents for the chemotherapeutic drug, 10-hydroxycamptothecin (HCPT). ${ }^{73}$ Similar to the previous study by Huang et al, ${ }^{72}$ NDs were able to adsorb the drug onto their surfaces via simple physical forces. ${ }^{73}$

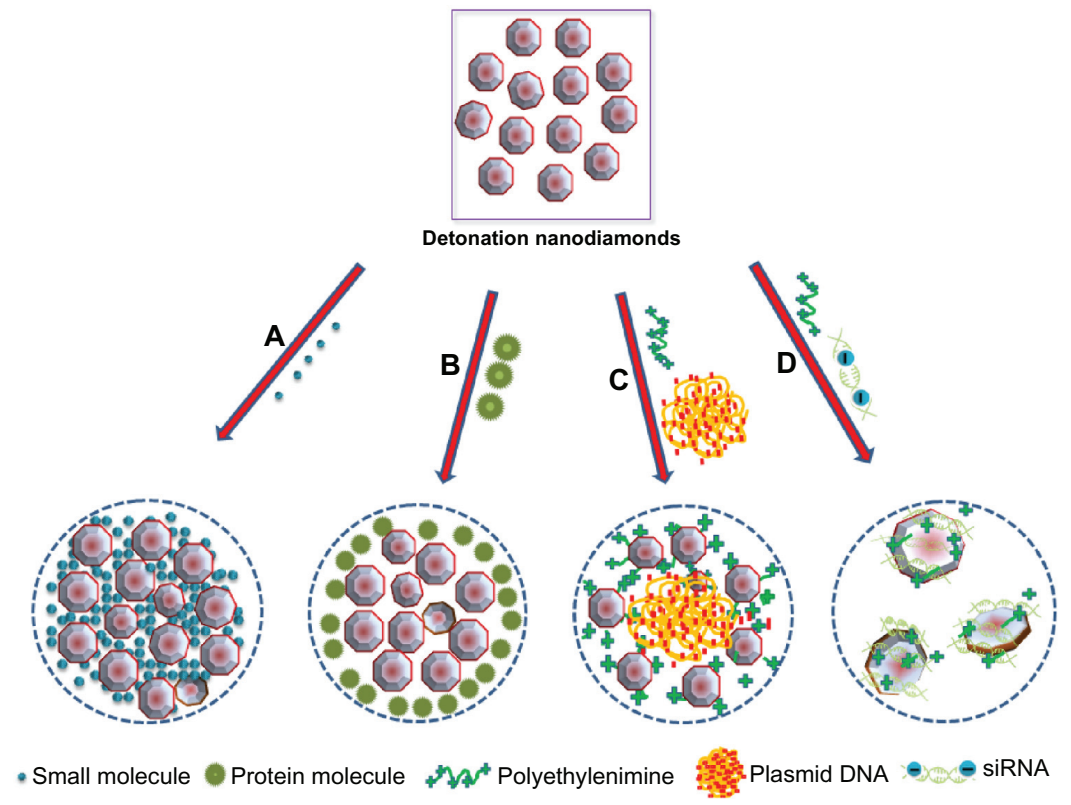

Figure 7 A schematic diagram representing the binding of detonation nanodiamonds with (A) small molecules, ${ }^{72,73}$ (B) proteins, ${ }^{73}$ (C) plasmid DNA, ${ }^{66,109}$ and (D) siRNA. ${ }^{110}$ C20 I 2 Dove Medical Press. Adapted with permission from Kaur R, Chitanda JM, Michel D, et al. Lysine-functionalized nanodiamonds: synthesis, physiochemical characterization, and nucleic acid binding studies. Int J Nanomedicine. 2012;7:385I-3866. 
However, in this case, the surface loading of HCPT was considerably enhanced to $50 \mathrm{wt} \%$ from $0.4 \mathrm{wt} \%$ with an increase in $\mathrm{pH}$ of the HCPT solution from 7 to 8.2 , rather than by means of salination. ${ }^{73}$ The NDs released HCPT slowly into the phosphate-buffered saline medium over a period of 5 days, with only $38 \%$ release observed in the first 24 hours. $^{73}$ In addition, the ND-HCPT complex showed almost 2.5 times higher cytotoxicity in HeLa cells than the chemotherapeutic activity of HCPT alone, which was credited to the ND-triggered intracellular delivery of HCPT. ${ }^{73}$ Bovine serum albumin adsorbed on the surface of NDs rapidly ( $<1$ hour) while HCPT showed a gradual adsorption ( $>120$ hours).${ }^{73}$ Based on this differential adsorption, a model suggesting porous clusters of NDs was developed in which small molecules diffuse inside the clusters (Figure 7A) and large molecules adsorb only on the outer surface of the NDs (Figure 7B).$^{73}$ Hence, NDs can facilitate the intracellular delivery of both small chemotherapeutic drugs and large therapeutic biomolecules. ${ }^{73}$

Detonation NDs have also been utilized to increase the aqueous dispersibility of hydrophobic drugs. Poorly water soluble chemotherapeutic drugs such as purvalanol A, with therapeutic activity against liver cancer, ${ }^{95}$ and 4-hydroxytamoxifen, with a high potential to treat breast cancer, ${ }^{96,97}$ are soluble in the polar, organic solvents DMSO and ethanol, respectively. ${ }^{92}$ The use of nonaqueous solvents limits the parenteral administration of these formulations in clinically relevant settings. However, when formulated with NDs, the aqueous dispersibility of purvalanol A and 4-hydroxytamoxifen was enhanced markedly. ${ }^{92}$ By adsorbing the drug on their surfaces, NDs considerably reduced particle size and increased the zeta potential of these drugs in water, promoting their dispersibility and, potentially, cellular uptake. ${ }^{92}$ Similar to the previous studies, the NDs preserved the therapeutic activity of the drugs, as demonstrated by DNA fragmentation and 3-(4,5-Dimethylthiazol2-yl)-2,5-diphenyltetrazolium bromide (MTT) assay for ND-4-hydroxytamoxifen and ND-purvalanol A composites, respectively. ${ }^{92}$ These findings suggest that NDs could play an important role in designing injectable formulations of water insoluble drugs.

The efficacy of NDs to deliver chemotherapeutic drugs was examined recently in animal models for the first time. ${ }^{85}$ In addition to generalized toxicity, large particle size, and poor water solubility, chemotherapeutic drugs also trigger acquired and intrinsic chemoresistance in tumor cells. ${ }^{98}$ Among various other factors, overexpression of P-glycoprotein on the cellular membranes, causing premature efflux of the drug from cells, is the leading cause for the development of chemoresistance in mammalian tumor cells. ${ }^{99,100}$ This challenge could be addressed by using a delivery system that not only enhances the uptake of the chemotherapeutic drugs but also retains them in the cancer cells for a longer period. The study revealed that DOX bound to the ND surface was significantly more toxic towards DOX-resistant mouse LT2-Myc liver and 4T1 mammary tumor models than bare DOX. ${ }^{85}$ The high chemotherapeutic efficacy of the ND-DOX composite was attributed to ND-mediated DOX retention in tumor cells as determined by fluorescence microscopy and quantitative analysis. ${ }^{85}$ Moreover, long-term treatment showed the superiority of the DOX bound to ND over the bare DOX in preventing tumor growth. ${ }^{85}$ NDs not only circumvented the premature efflux of DOX from tumor cells but also improved the adverse effects of naked DOX by significantly reducing myelosuppression and early mortality. ${ }^{85}$ In addition, NDs increased the circulation half time of DOX from 0.83 to 8.43 hours, which further validated their sequestering behavior proposed in earlier studies. ${ }^{72,73,85}$

In summary, formulation of chemotherapeutic drugs with NDs improves the pharmaceutical properties of the agents by providing high surface loading, improved aqueous dispersibility, sustained tunable release, and enhanced retention in chemoresistant cells. These properties are due to the hydrophilic functional group-enriched surface, large surface-to-volume ratio, ability to form loose clusters, improved cellular delivery, and biocompatibility of the NDs. Hence, NDs are suitable platforms on which to build nanoparticles for overcoming some of the major deficiencies of the chemotherapeutic drugs.

In addition to small molecules, NDs have shown potential as delivery agents for protein, DNA, and RNA.

\section{Delivery of biotechnology products}

The potential of NDs as a targeted protein-delivery vehicle was evaluated in a $\mathrm{pH}$-dependent system. ${ }^{101}$ By means of physical adsorption, NDs achieved a considerably high surface loading of bovine insulin $(-80 \%)$ in $\mathrm{pH}$-neutral water at a weight ratio of 1:4 of insulin:NDs. ${ }^{101}$ Similar to a previous study, ${ }^{92}$ the aggregation properties (size, zeta potential and polydispersity index) of the insulin improved after interacting with NDs. ${ }^{101}$ This suggests that NDs have the ability to facilitate uniform-sized complex formation. ${ }^{101}$ Further, the release of insulin from the surface of the NDs was almost 20 times higher when at a $\mathrm{pH}$ of 10.5 than when in a neutral $\mathrm{pH}$ medium. ${ }^{101}$ The applicability of ND-bound insulin was verified 
by MTT assay in serum-starved murine macrophages. ${ }^{101}$ A higher cellular viability was observed with sodium hydroxide-treated ND-insulin than with neutral $\mathrm{pH}$-treated ND-insulin. ${ }^{101}$ Similar results were obtained by measuring the gene expression of insulin 1 and granulocyte colony stimulating factor in serum-starved mouse adipocytes. ${ }^{101}$ Hence, formulations of insulin can be designed with NDs to target the recovery of injured tissues ${ }^{102}$ that have a basic $\mathrm{pH}$ due to bacterial growth. ${ }^{101,103}$

Although there is compelling evidence to support the utilization of NDs as drug delivery agents for small molecules, their potential as gene delivery vectors has been less widely explored. The major obstacle to the use of naked nucleic acids as therapeutics is their inefficient cellular delivery. Physicochemical characteristics such as high negative surface charge and large molecular weight are barriers in the efficient cellular internalization of the DNA ${ }^{104,105}$ and small interfering RNA (siRNA). ${ }^{106,107}$ While DNA is too large for cellular uptake, the relatively small size of siRNA also poses an additional challenge to its cellular delivery, as particles having a molecular weight of less than $50 \mathrm{kDa}$ are susceptible to excretion through glomerular filtration. ${ }^{106,108}$ Zhang et al investigated NDs as a platform on which to build enhanced plasmid DNA (pDNA) delivery systems (Figure 7C). ${ }^{109}$ At first, they coated NDs with polyethyleneimine 800 (PEI800) then allowed them to interact with luciferase pDNA via electrostatic forces. ${ }^{109}$ NDs were found to enhance the transfection efficiency of the polymer by 70 -fold at a $15: 1$ weight ratio of ND-PEI800:pDNA in HeLa cells, which might be due to ND-mediated cellular and nuclear uptake of the pDNA. ${ }^{109}$ Similar to DNA delivery, the ND-based complex (ND-PEI800) was used to deliver anti-green fluorescent protein siRNA to breast cancer cells expressing green fluorescent protein (Figure 7D). ${ }^{110}$ Compared with the PEI-siRNA, the ND-PEI800:siRNA complex at a weight ratio of 3:1 showed an almost twofold higher knockdown in cellular green fluorescent protein expression. ${ }^{110}$ In the presence of serum in the treatment medium, the ND-PEI800:siRNA complex showed better knockdown in green fluorescent protein expression as well as lower cytotoxicity than Lipofectamine ${ }^{\mathrm{TM}}$, which is a gold standard for in vitro delivery of nucleic acids. ${ }^{110}$ Therefore, NDs have the potential to enhance the transfection ability of polymers while remaining biocompatible with the cell lines.

However, so far, there have been limited studies of the applications of NDs as drug delivery agents. More studies are needed to verify their potential and to translate their use as a delivery agent into clinical applications.

\section{NDs as bioimaging agents Fluorescence of the NDs}

Based upon the presence or lack of nitrogen impurities in the crystal lattice structure, the natural diamonds are classified as type I or type II, respectively. ${ }^{111}$ The arrangement of nitrogen impurities is used to subclassify type I diamonds into type Ia and type $\mathrm{Ib} .{ }^{112}$ While type $\mathrm{Ib}$ contain atomically dispersed, single substitutional nitrogen impurities (C-centers), type Ia diamonds comprise an aggregated form of nitrogen. ${ }^{112}$ The latter are categorized further into type IaA, having two aggregated nitrogen atoms (A-center); type $\mathrm{IaB}$, having platelets and four aggregated nitrogen atoms surrounding the nearest lattice vacancy (B-center); and type IaA/B, possessing the characteristics of both type IaA and type IaB. ${ }^{112}$

Besides natural diamonds, synthetic diamonds manufactured in high-pressure high-temperature conditions are also known to contain a high amount of nitrogen in the form of impurities. ${ }^{113}$ The natural diamonds have mainly aggregated nitrogen defects (type Ia), while synthetic high-pressure hightemperature diamonds are predominantly embellished with single substitutional nitrogen centers (type Ib). ${ }^{114}$ Irradiation damage of type Ib diamond crystals creates intrinsic defects, such as vacancies, which, upon thermal annealing, move towards nitrogen centers $(\mathrm{N})$ and become trapped within to form N-V color defect centers. ${ }^{23}$ Generally, irradiation of these diamond particles is carried out by high-energy electron $(\sim 2 \mathrm{MeV})^{115-117}$ or proton $(\sim 3 \mathrm{MeV})^{69,118}$ beam using Van de Graaff or tandem particle accelerators, respectively. These sources generate vacancies but are difficult to install in usual pharmaceutical or biomedical laboratory settings due to their high cost, complicated setup, and safety issues. ${ }^{19}$ Hence, to increase the feasibility of the wider production of fluorescent NDs, a moderate scale-energy helium-ion $(40 \mathrm{keV})$ beam emanating from a radio frequency positiveion source was proposed. ${ }^{19}$ Even though a much lower energy source was used in this irradiation process, none of the essential fluorescence spectral features were lost, apart from a decrease in fluorescence intensity, when compared with the fluorescence spectra generated by $3 \mathrm{MeV}$ energy proton beam-irradiated particles. ${ }^{19}$ This method not only benefited the laboratories having ordinary infrastructure in producing fluorescent NDs but also increased the yield of fluorescent NDs by creating a higher number of vacancies compared with electron beam- and proton beam-irradiated NDs. ${ }^{19,118,119}$

After 2 hours of annealing at $800^{\circ} \mathrm{C}$, irradiated synthetic type Ib NDs mainly generated two types of N-V defect 
centers: neutral nitrogen vacancy $(\mathrm{N}-\mathrm{V})^{0}$, with zero phonon line at $575 \mathrm{~nm}$, and negatively charged nitrogen vacancy $(\mathrm{N}-\mathrm{V})^{-}$, with zero phonon line at $\sim 638 \mathrm{~nm} .{ }^{19,88,116}$ Among all the color centers, $(\mathrm{N}-\mathrm{V})^{-}$dominate the fluorescence spectra of type $\mathrm{Ib}$ diamonds. ${ }^{23,117}$ This defect center absorbs light photons at $\sim 560 \mathrm{~nm}$ and fluoresces brightly at $\sim 700 \mathrm{~nm}$ with a quantum efficiency close to $1 .{ }^{25,69,120}$ As such, these centers offer characteristically bright red fluorescence to diamond particles upon excitation with green-yellow light, ${ }^{22}$ hence have found applications for high-pressure high-temperature NDs in imaging studies. ${ }^{19,25}$

The extreme photostability of $(\mathrm{N}-\mathrm{V})^{-}$defect centers ${ }^{121,122}$ provides a greater advantage to NDs to emerge as an excellent candidate for long-term cellular imaging over commonly used fluorophores. Upon continuous exposure to a $100 \mathrm{~W}$ lamp power for 480 minutes, the red fluorescent NDs $(100 \mathrm{~nm})$ remained stable with no observed photobleaching whereas similarly sized red fluorescent polystyrene nanospheres photobleached within the first 30 minutes of photoexcitation. ${ }^{69}$ In another instance, no photobleaching occurred in both 35 and $100 \mathrm{~nm}$ red fluorescent NDs when photoexcited with $8 \mathrm{~kW} / \mathrm{cm}^{2}$ power density of light for 5 minutes. ${ }^{25}$ In contrast, under the same photoexcitation conditions, the fluorescence of Alexa Fluor ${ }^{\circledR} 546$ dye conjugated to DNA faded completely in 12 seconds. ${ }^{25}$ Additionally, the fluorescence of red NDs was found to be significantly brighter than that emitted by Alexa Fluor $546 .{ }^{25}$ The evidence of photostability has also been determined for a single $7 \mathrm{~nm}$ red fluorescent diamond nanocrystal that did not show any sign of photobleaching over 30 seconds. ${ }^{123}$ In addition to resistance to photobleaching, the fluorescence of NDs (35 and $100 \mathrm{~nm}$ in size) was also found to be immune to photoblinking on a time scale of 1 millisecond. ${ }^{25}$ Moreover, due to the deep location of $(\mathrm{N}-\mathrm{V})^{-}$centers in the lattice structure of NDs, ${ }^{124,125}$ the fluorescence remains unaffected by surface alterations, as evidenced by NDs functionalized with carboxylic acid, ${ }^{69}$ polylysine,${ }^{25}$ and transferrin ${ }^{126}$ groups . Therefore, the intrinsic photostability of NDs opens avenues for carrying out chemical modification of their surface for specific imaging applications.

Furthermore, the red fluorescent NDs could be more advantageous than organic fluorophores in obtaining bright contrast images in cells. The absorption and emission wavelength of cellular endogenous components such as flavins, collagens, lipofuscins, and coenzymes, ranging from $\sim 280$ to $630 \mathrm{~nm},{ }^{127,128}$ coincides with most of the organic fluorophores used for cellular imaging. ${ }^{129}$ Thus, it provides an undesirable, strong background to the fluorescence signal.
However, fluorescent NDs can act as an ideal candidate in this case, as emission of the dominant color center of NDs, $(\mathrm{N}-\mathrm{V})^{-}$, occurs above $690 \mathrm{~nm},{ }^{25,69}$ well separated from the cellular autofluorescence region. In addition, the average fluorescence lifetime of NDs is 17 nanoseconds, ${ }^{25,123}$ which is considerably longer than the lifetime of fluorescent organic dyes $(<5 \text { nanoseconds })^{130}$ and most of the cellular endogenous fluorophores ( $<6$ nanoseconds). ${ }^{127}$ Hence, by time gating, ${ }^{131}$ it is possible to collect the fluorescence of the NDs alone with minimal background cellular autofluorescence, thereby providing high-contrast imaging of NDs in the cells, which is not feasible with most of the commonly used organic fluorophores. ${ }^{25}$ Table 1 lists the advantages of using red fluorescent NDs as a fluorescent tag for in vitro or in vivo tracking applications.

Similar to the N-V defect centers of type Ib synthetic diamonds, the nitrogen-vacancy-nitrogen $(\mathrm{N}-\mathrm{V}-\mathrm{N})$ or $\mathrm{H} 3$ defect centers ${ }^{24,132}$ in type Ia diamond nanocrystals can be created by irradiating them with a high-energy $(3 \mathrm{MeV})$ proton or a medium-energy $(40 \mathrm{keV})$ helium-ion beam followed by annealing at $800^{\circ} \mathrm{C} .{ }^{87} \mathrm{H} 3$ defect centers have a zero phonon line at $503 \mathrm{~nm}$, with absorption and fluorescence emission at 470 and $530 \mathrm{~nm}$, respectively. ${ }^{22}$ Therefore, type Ia NDs with $\mathrm{H} 3$ centers are known as "green fluorescent NDs."

Other than the natural and high-pressure high-temperature NDs containing nitrogen impurities, researchers are also trying to understand the fluorescence characteristics of detonation NDs. Considering the actual composition of the explosive mixture ( 20 mass $\%$ of nitrogen) used to synthesize these particles, they might contain a high concentration of nitrogen, ${ }^{133}$ either in their core structure or in the form of surface defects. Initially, these nanoparticles were suggested to be devoid of $\mathrm{C}$-centers and believed to be unsuitable for imaging applications..$^{21}$ The absence of zero phonon lines corresponding to the N-V centers in irradiated and annealed detonation NDs also suggested that structural defects were the origin of their fluorescence. ${ }^{134} \mathrm{Z}$-contrast scanning transmission microscopy,

Table I Highlights of the unique fluorescence properties of nanodiamonds

\begin{tabular}{ll}
\hline Property & Advantage \\
\hline High quantum efficiency & Bright fluorescence \\
Photostability over longer & Long-term tracking of nanodiamonds \\
periods & in cells \\
Emission wavelength $>690 \mathrm{~nm}$ & High-contrast imaging of nanodiamonds \\
Longer fluorescence lifetime & High-contrast imaging of nanodiamonds \\
Deeper location of nitrogen & No alteration in fluorescence due to \\
vacancy centers & surface modification \\
\hline
\end{tabular}


high-resolution transmission electron microscopy, and electron energy loss spectroscopy identified nitrogen in the core structure of detonation NDs. ${ }^{135}$ Recently, Vlasov et al detected stable N-V color centers in detonation NDs greater than $30 \mathrm{~nm}$ after their irradiation and thermal annealing treatment. ${ }^{133}$ While the occurrence of $\mathrm{N}-\mathrm{V}$ color centers in the bulk structure of detonation NDs of less than $10 \mathrm{~nm}$ has been questioned in various studies, ${ }^{136,137}$ Smith et al showed the presence of these centers in single-digit-sized irradiation-damaged and annealed NDs. ${ }^{138}$ Moreover, N-V color centers have also been discovered in nonirradiated $5 \mathrm{~nm}$ detonation NDs. ${ }^{139}$ The NDs synthesized by the detonation technique are shown to possess up to 3 at $\%$ of nitrogen impurities. ${ }^{133,140}$

NDs, due to their bright fluorescence, high photostability with sufficiently long lifetime and excellent biocompatibility, are emerging as efficient and safe candidates for cellular imaging.

In 2007, Fu et al explored the fluorescent properties of a single $100 \mathrm{~nm}$ red fluorescent ND to examine the binding pattern of negatively charged DNA with a positively charged ND. ${ }^{25}$ First, the molecules of T4 DNA labeled with TOTO-1 fluorophore were allowed to interact electrostatically with polyL-lysine-coated carboxylated fluorescent NDs in a buffer. ${ }^{25}$ The diluted solution containing a single fluorescent ND and DNA was then added into a microchannel of a coverglass plate terminated with amino groups, followed by DNA stretching using microchannel-combing methodology. ${ }^{25}$ After laser excitation of the sample at $514 \mathrm{~nm}$, fluorescence was detected using a wide-field epifluorescence microscope equipped with 545 to $605 \mathrm{~nm}$ and 675 to $685 \mathrm{~nm}$ wavelength channels. ${ }^{25}$ By overlapping the fluorescence emitted from the TOTO-1 dye and the ND, detected by the shorter wavelength channel, with that of ND alone, detected by the longer wavelength channel, it was discovered that the DNA interacts with the positively charged single ND through a wrapping arrangement (Figure 8). ${ }^{25}$ Furthermore, by using the fluorescence intensity of the ND as a control, it was observed that over a period of 13.9 seconds, a $35 \mathrm{~nm}$ ND travels within a $1 \times 1 \mu^{2}$ area close to the nucleus of HeLa cells. ${ }^{25}$ To compare the photostability of TOTO-1 dye with fluorescent ND, samples were excited for 40 seconds at $514 \mathrm{~nm} .{ }^{25}$ No sign of photobleaching was observed in the ND, whereas the dye started fading within the first 5 seconds. ${ }^{25}$ This study shows that a single fluorescent ND can function as tracking agent and probe to investigate the interaction between biomolecules.

In 2009, Weng et al investigated the uptake mechanism of transferrin in HeLa cells using the fluorescent properties of NDs as a biomolecule label. ${ }^{126}$ They oxidized $100 \mathrm{~nm}$ red fluorescent NDs and conjugated transferrin to their surfaces through an amide linkage to form ND-transferrin conjugates. ${ }^{126}$ These bioconjugates were then incubated for an hour at a concentration of $10 \mu \mathrm{g} / \mathrm{mL}$ with HeLa cells overexpressing transferrin receptors. ${ }^{126}$ To further verify the findings, ND-transferrin complexes were added to HeLa cells in which their transferrin receptors were pre-saturated by adding an excess of transferrin. ${ }^{126}$ Upon excitation of the treated HeLa cell samples using a $514.5 \mathrm{~nm}$ laser source, confocal fluorescence images were obtained by collecting fluorescence from 663 to $738 \mathrm{~nm}$, thereby excluding most of the cellular autofluorescence background and providing high-contrast images of NDs in cells (Figure 9). ${ }^{126}$ While the successful internalization of transferrin was observed in the HeLa cells overexpressing transferrin receptors, no noticeable uptake occurred in transferrin-receptor pre-saturated HeLa cells, as evidenced by the absence of ND fluorescence emission in the latter. ${ }^{126}$ Hence, by using the fluorescence of NDs as a marker, it was confirmed that uptake of transferrin in HeLa cells occurs through receptor-mediated endocytosis. ${ }^{126}$ Moreover, $100 \mathrm{~nm}$ NDs did not interfere in the binding of transferrin to its receptor and subsequent cellular uptake. ${ }^{126}$ Moreover, it was verified
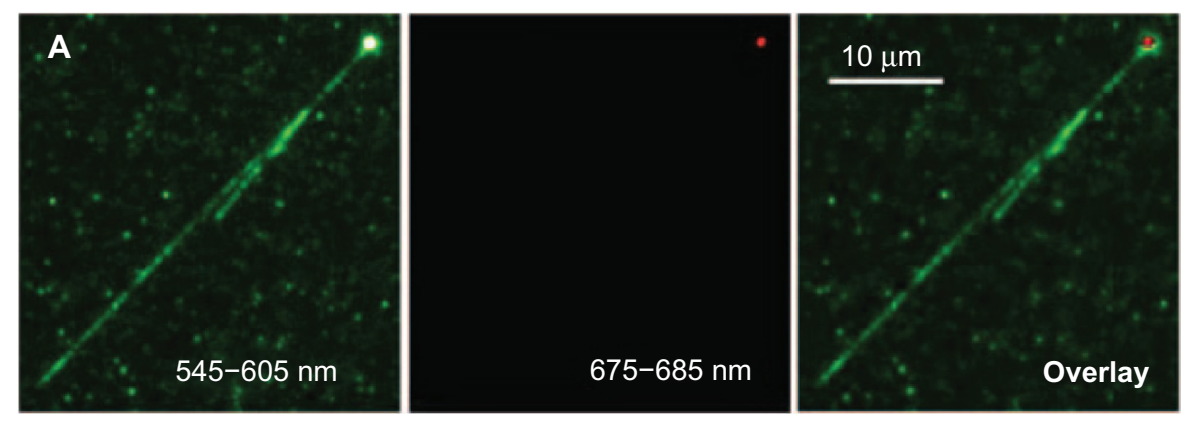

Figure 8 Overlay image (far right) demonstrating the wrapping of TOTO-I dye-labeled T4DNA (green color, V-shape) around the surface of a single polyL-lysine-coated fluorescent nanodiamond (red color).

Reprinted with permission from: Fu CC, Lee HY, Chen K, et al. Characterization and application of single fluorescent nanodiamonds as cellular biomarkers. Proc Natl Acad Sci U S A. 2007; 104(3):727-732. ${ }^{25}$ Copyright (2007) National Academy of Sciences, USA. 

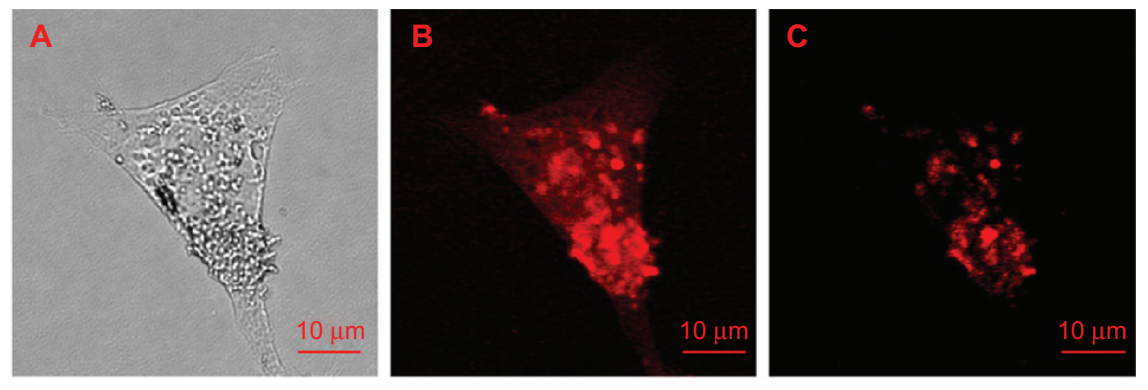

Figure 9 Images of a single HeLa cell treated with ND-transferrin complex: (A) bright-field image, (B) confocal scanning image obtained by collecting all the fluorescence emissions above a wavelength of $550 \mathrm{~nm}$, and $(\mathbf{C})$ confocal scanning image obtained by collecting only the fluorescence emissions at wavelengths of $663-738 \mathrm{~nm}$. Note: A $514.5 \mathrm{~nm}$ laser was used as excitation source.

Reprinted from: Weng MF, Chiang SY, Wang NS, Niu H. Fluorescent nanodiamonds for specifically targeted bioimaging: application to the interaction of transferrin with transferrin receptor. Diam Relat Mater. 2009;18(2-3):587-591. ${ }^{126}$ Copyright (2008), with permission from Elsevier.

Abbreviation: ND, nanodiamonds.

that the fluorescence of the NDs originates from the lattice color defect center rather than surface defects, as the surface conjugation of NDs with transferrin and the transferrin-NDtransferrin receptor interactions had no adverse effects on their fluorescence properties. ${ }^{126}$ Hence, this result shows that NDs can act as an efficient label to examine the cellular uptake mechanism of various biomolecules.

In addition to the various explorations of the red fluorescence of type Ib irradiated/annealed synthetic NDs as cellular biomarkers, ${ }^{25,115,126}$ in 2009, Mkandawire et al proposed the use of untreated detonation NDs, emitting green fluorescence, for labeling live cells. ${ }^{141}$ Immunoconjugated ND complexes were prepared by covalently coupling actin and mitochondrial-targeted antibodies to the surface of oxidized NDs. ${ }^{141}$ The actin antibody-ND and mitochondria antibody-ND immunoconjugates were then allowed internalization by HeLa cells using maltotriose-shelled fourthgeneration dendrimers. ${ }^{141}$ Using the green fluorescence of the NDs as a marker, live-cell fluorescence microscopic images of the treated cells, collected at 535/40 nm wavelength, revealed selective targeting of actin and mitochondria by actin antibody-ND and mitochondria antibody-ND immunoconjugates, respectively. ${ }^{141}$ With the rational selection of an antibody, NDs could also be designed to image the specific structures of the cells. ${ }^{141}$

Further to the in vitro studies, in 2010, Mohan et al traced the movement of bare and bioconjugated red fluorescent NDs in Caenorhabditis elegans (Figure 10), a hermaphrodite with an optically transparent body. ${ }^{142}$ The worms were deprived of Escherichia coli, and then fed bare and dextran-/bovine serum albumin-conjugated carboxylated NDs. ${ }^{142}$ The location of NDs in the worms was determined by differential interference and wide-field epifluorescence microscopic images, obtained by collecting the emission from the sample above a wavelength of $600 \mathrm{~nm}$ upon excitation with green-yellow light $(510-560 \mathrm{~nm}){ }^{142}$ The bare NDs were found to be localized in the lumen of the worm 12 hours after administration, with no signs of intestinal absorption. ${ }^{142}$ However, the excretion of these NDs took place within an hour when the worms were fed E. coli. ${ }^{142}$ In contrast to the bare NDs, a major fraction of the bioconjugated NDs was taken up by the intestinal cells of the worm and remained there for 24 hours, despite $E$.coli feeding. ${ }^{142}$ The high intestinal absorption of bioconjugated NDs compared with bare NDs was believed to be due to enhanced dispersibility of the NDs as a result of surface functionalization and/or triggering of certain cellular uptake pathways. ${ }^{142}$ No photobleaching or photoblinking of NDs was observed, even after 48 hours of continuous excitation with light. ${ }^{142}$ Furthermore, observation of the lifespan, brood size, reactive oxygen species, and stress response level demonstrated high biocompatibility of the NDs. ${ }^{142}$ This study shows the potential for the use of the fluorescent properties of NDs in long-term tracking of biomolecules in organisms and as tools for marking human organelles. ${ }^{142}$

To date, there are a number of publications showing the utilization of ND fluorescence for imaging, mostly in the cells and less commonly in translucent organisms. However, further studies need to be carried out to demonstrate the usefulness of NDs as imaging agents in animals and humans. Imaging organisms that do not possess translucent membranes could pose a challenge.

\section{Elastic and inelastic light scattering imaging}

In addition to the fluorescence, NDs have a refractive index of 2.42, thus possess characteristic optical scattering properties. ${ }^{124}$ This property was explored by Smith et al by obtaining differential interference contrast microscopic images of $55 \mathrm{~nm}$ NDs internalized into 3T3 mammalian 
I

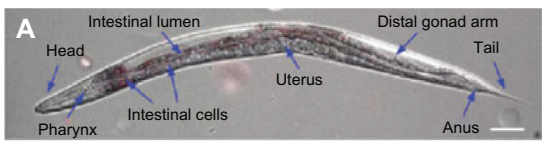

B
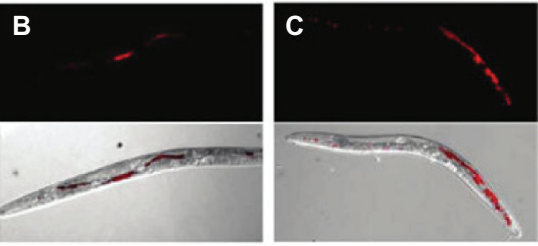

D

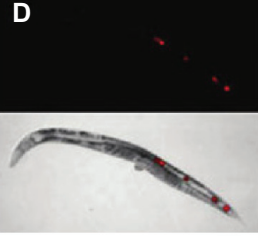

II

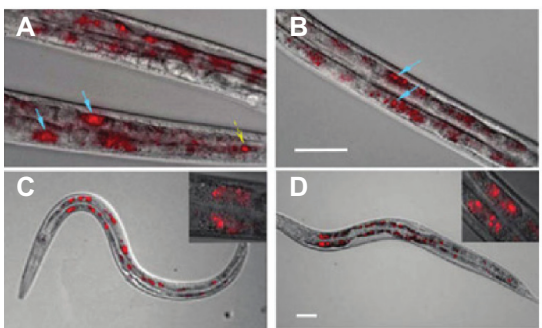

Figure 10 (I) Images of Caenorhabditis elegans: (A) an untreated worm with labeled organ morphology; worms after oral administration of bare nanodiamonds (NDs) for (B) 2 hours and (C) 12 hours; worms fed with bare NDs for 2 hours with subsequent administration of Escherichia coli for (D) 20 minutes (E) and 40 minutes. (B-E) upper show epifluorescence images; lower show epifluorescence and differential interference contrast merged images. (II) Epifluorescence and differential interference contrast merged images of Caenorhabditis elegans: worms after oral administration of (A) dextran-conjugated NDs, (B) bovine serum albuminconjugated NDs, and worms exposed to Escherichia Coli after administration of (C) dextran-conjugated NDs and (D) bovine serum albumin-conjugated NDs.

Notes: Blue arrows indicate internalization of NDs in intestinal cells; yellow arrow indicate localization of NDs in lumen of worm.

Reprinted with permission from: Mohan N, Chen CS, Hsieh HH, Wu YC, Chang $\mathrm{HC}$. In vivo imaging and toxicity assessments of fluorescent nanodiamonds in Caenorhabditis elegans. Nano Lett. 2010;10(9):3692-3699. ${ }^{142}$ Copyright (2010) American Chemical Society.

epithelial cells. ${ }^{143}$ Based upon the differences in refractive indices, the Rayleigh light scattering of diamond nanocrystals is 300 times higher compared to cellular structures of the same size. ${ }^{144}$ This offers an advantage in using NDs for obtaining high-contrast imaging in the cells. In addition to elastic scattering, NDs are also capable of scattering light inelastically, hence are Raman active. ${ }^{145,146}$ The phonon mode of the diamond carbon shows a sharp and distinguished Raman signal at $1332 \mathrm{~cm}^{-1},{ }^{147}$ providing an opportunity for spectroscopic characterization and mapping of NDs in the cells. Raman spectroscopy is a noninvasive and nontoxic technique, therefore has the potential to be used for the detection and imaging of NDs in biological samples. Also, Raman spectroscopic measurements do not require complicated sample preparation and conditions that are hard to maintain, such as vacuum. Therefore, in addition to the fluorescent tag, NDs can also be used as an optical scattering nanoprobe in cellular studies.

In 2007, Perevedentseva et al used NDs as nanobioprobes to observe biomolecule-bacterial interactions in vivo, using the unique Raman signal of NDs as a detection marker. ${ }^{145}$ Physical adsorption of lysozymes was performed on carboxylated NDs and the resultant carboxylated ND-lysozyme complex was then allowed to interact with E. coli. ${ }^{145}$ The optical microscope located the E. coli, while the confocal Raman spectrometer mapped the Raman signals from the NDs. ${ }^{145}$ As the lysozyme was adsorbed onto the surface of the NDs, the overlay of optical and Raman images revealed the interaction sites of the lysozyme with $E$.coli (Figure 11). ${ }^{145}$ This dual imaging provided a simple method through which to observe the interaction of molecules with living cells. ${ }^{145}$ Furthermore, there was no reduction in the bactericidal activity of lysozymes upon adsorption onto the NDs' surface, proving that the NDs did not interfere with the therapeutic activity of the attached entity. ${ }^{145}$

In 2007, the same group investigated the use of NDs to predict the location of growth-hormone receptors in human lung

I. cND + E.coli II. cND - LYS + E.coli

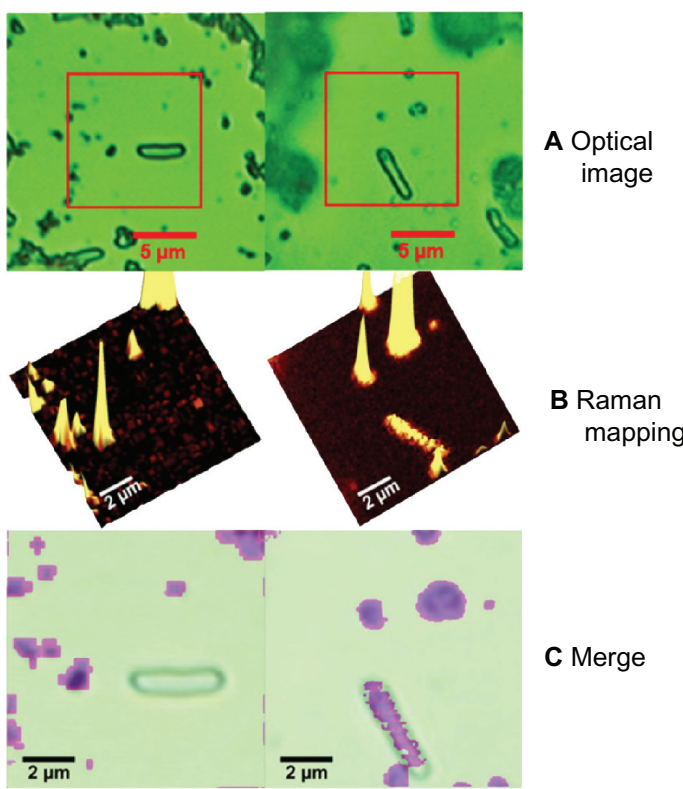

Figure I I Lysozymal interaction sites revealed with Escherichia coli: (I) carboxylated nanodiamonds (NDs) and Escherichia coli, (II) carboxylated ND-lysozyme complex and Escherichia coli. (A) Optical images identifying Escherichia coli; (B) confocal Raman mapping identifying NDs present in the square area outlined in optical image; (C) merging of the optical image with Raman mapping, demonstrating the interaction of the lysozymes with Escherichia coli. ${ }^{145}$

Reproduced from: Perevedentseva E, Cheng CY, Chung PH, Tu JS, Hsieh YH, Cheng $\mathrm{CL}$. The interaction of the protein lysozyme with bacteria $\mathrm{E}$. coli observed using nanodiamond labelling. Nanotechnology. 2007; 18(31):315102, ${ }^{145}$ courtesy of IOP Publishing Ltd. 
cancer cells by identifying the $\mathrm{sp}^{3}$ carbon Raman signals of NDs. ${ }^{146}$ Growth hormone-ND complexes were created by carboxylating and covalently linking $100 \mathrm{~nm}$ NDs to the growth hormone. ${ }^{146}$ When mapped by confocal Raman spectrophotometry, the NDs bound to growth hormone were located on the surface of cells, while the unconjugated carboxylated NDs were internalized into the cells. ${ }^{146}$ These observations verified the extracellular location of the growth-hormone receptor using the Raman signal of the ND as a label. ${ }^{146}$ This study provided a relatively easy and direct method of locating receptors at the cellular level. This method could be beneficial in investigating the receptor-mediated internalization of biomolecules and diagnosing diseases such as cancers involving the alteration in expression of certain receptors. ${ }^{146}$

\section{Conclusion}

Continued investigation and understanding of disaggregation methods, surface properties, functionalization, biocompatibility, cellular fate, fluorescence, and the optical scattering characteristics of NDs have opened new horizons for their applications in the biomedical field. NDs have shown great potential to emerge as a platform for delivering drugs into biological systems due to their biocompatibility, high loading capacity, and ability to cross cellular membranes. These particles have imparted sustained-release and hydrophilic characteristics to water insoluble drugs, and have opened up the possibilities for systemic administration of highly effective, hydrophobic chemotherapeutic molecules. While N-V defect centers are responsible for conferring fluorescence to NDs, the scattering properties originate from their high refractive index and Raman optical activity. These properties provide imaging capabilities to NDs for their detection in the cell. The multifunctional characteristics of NDs can be utilized to improve the delivery of drugs in conjunction with imaging their fate in biological systems, which can revolutionize studies in the area of life sciences. This unique biomaterial could be further explored as a gene delivery vehicle and a label to identify the biomolecular targets of drugs.

\section{Acknowledgments}

The authors thank Dr Ramaswami Sammynaiken (Saskatchewan Structural Sciences Centre, University of Saskatchewan) and Dr Ronald E Verrall (Department of Chemistry, University of Saskatchewan) for their valuable suggestions for improving the manuscript.

\section{Disclosure}

The authors report no conflicts of interest in this work.

\section{References}

1. Shenderova OA, McGuire G. Types of nanocrystalline diamond. In: Shenderova OA, Gruen DM, editors. Ultrananocrystalline Diamond: Synthesis, Properties, and Applications. New York, NY: William Andrew; 2006:79-114.

2. Mochalin VN, Shenderova O, Ho D, Gogotsi Y. The properties and applications of nanodiamonds. Nat Nanotechnol. 2012;7(1):11-23.

3. Gracio JJ, Fan QH, Madaleno JC. Diamond growth by chemical vapour deposition. J Phys D Appl Phys. 2010;43:374017.

4. Philip J, Hess P, Feygelson T, et al. Elastic, mechanical, and thermal properties of nanocrystalline diamond films. J Appl Phys. 2003;93(4): 2164-2171. Available at: http://dx.doi.org/10.1063/1.1537465.

5. Zhou D, Gruen DM, Qin LC, McCauley TG, Krauss AR. Control of diamond film microstructure by Ar additions to $\mathrm{CH} 4 / \mathrm{H} 2$ microwave plasmas. J Appl Phys. 1998;84(4):1981-1989.

6. Qin LC, Zhou D, Krauss AR, Gruen DM. TEM characterization of nanodiamond thin films. Nanostructured Materials. 1998;10(4): 649-660.

7. Espinosa HD, Peng B, Prorok BC, et al. Fracture strength of ultrananocrystalline diamond thin films - identification of Weibull parameters. J App Phys. 2003;94(9):6076-6084.

8. Angadi MA, Watanabe T, Bodapati A, et al. Thermal transport and grain boundary conductance in ultrananocrystalline diamond thin films. J Appl Phys. 2006;99(11):114301-114301-6.

9. Butler JE, Sumant AV. The CVD of nanodiamond materials. Chemical Vapor Deposition. 2008;14(7-8):145-160.

10. Sumant AV, Grierson DS, Gerbi JE, Carlisle JA, Auciello O, Carpick RW. Surface chemistry and bonding configuration of ultrananocrystalline diamond surfaces and their effects on nanotribological properties. Phys Rev B Condens Matter Mater Phys. 2007;76(23):235429-1-235429-11. Available from http://link.aps.org/ doi/10.1103/PhysRevB.76.235429. Accessed 24 August 2012.

11. Bajaj P, Akin D, Gupta A, et al. Ultrananocrystalline diamond film as an optimal cell interface for biomedical applications. Biomed Microdevices. 2007;9(6):787-794.

12. Shi B, Jin Q, Chen L, Auciello O. Fundamentals of ultrananocrystalline diamond (UNCD) thin films as biomaterials for developmental biology: Embryonic fibroblasts growth on the surface of (UNCD) films. Diam Relat Mater. 2009;18(2-3):596-600.

13. Krauss AR, Auciello O, Gruen DM, et al. Ultrananocrystalline diamond thin films for MEMS and moving mechanical assembly devices. Diam Relat Mater. 2001;10(11):1952-1961.

14. Auciello O, Birrell J, Carlisle JA, et al. Materials science and fabrication processes for a new MEMS technology based on ultrananocrystalline diamond thin films. J Phys Condens Matter. 2004; 16(16):R539-R552.

15. Auciello O, Sumant AV. Status review of the science and technology of ultrananocrystalline diamond (UNCD ${ }^{\mathrm{TM}}$ ) films and application to multifunctional devices. Diam Relat Mater. 2010;19(7-9):699-718.

16. Sumant AV, Auciello O, Carpick RW, Srinivasan S, Butler JE. Ultrananocrystalline and nanocrystalline diamond thin films for MEMS/ NEMS applications. MRS Bull. 2010;35(4):281-288. Available from: http://journals.cambridge.org/action/displayAbstract?fromPage $=$ onlin e\&aid $=7944009$.

17. Hupert M, Muck A, Wang J, et al. Conductive diamond thin-films in electrochemistry. Diam Relat Mater. 2003;12(10-11):1940-1949.

18. Yang W, Auciello O, Butler JE, et al. DNA-modified nanocrystalline diamond thin-films as stable, biologically active substrates. Nat Mater. 2002;1(4):253-257.

19. Chang YR, Lee HY, Chen K, et al. Mass production and dynamic imaging of fluorescent nanodiamonds. Nat Nanotechnol. 2008;3(5):284-288.

20. Sonnefraud Y, Cuche A, Faklaris O, et al. Diamond nanocrystals hosting single nitrogen-vacancy color centers sorted by photon-correlation near-field microscopy. Opt Lett. 2008;33(6):611-613.

21. Boudou JP, Curmi PA, Jelezko F, et al. High yield fabrication of fluorescent nanodiamonds. Nanotechnology. 2009;20(23):235602.

22. Chang HC. Development and use of fluorescent nanodiamonds as cellular marker. In: Ho D, editor. Nanodiamonds: Applications in Biology and Nanoscale Medicine. New York, NY: Springer; 2009:127-150. 
23. Davies G, Lawson SC, Collins AT, Mainwood A, Sharp SJ. Vacancyrelated centers in diamond. Phys Rev B Condens Matter. 1992;46(20): 13157-13170.

24. Clark CD, Norris CA. Photoluminescence associated with the 1.673, 1.944 and $2.498 \mathrm{eV}$ centres in diamond. Journal of Physics: C Solid State Phys. 1971;4(14):2223-2229.

25. Fu CC, Lee HY, Chen K, et al. Characterization and application of single fluorescent nanodiamonds as cellular biomarkers. Proc Natl Acad Sci U S A. 2007;104(3):727-732. DOI:10.1073/pnas.0605409104.

26. Dolmatov VY. Detonation synthesis ultradispersed diamonds: properties and applications. Russian Chemical Reviews. 2001;70(7):607-626.

27. Dolmatov VY. Synthesis and post-synthesis treatment of detonation nanodiamonds. In: Shenderova OA, Gruen DM, editors. Ultrananocrystalline Diamond: Synthesis, Properties, and Applications. New York, NY: William Andrew; 2006:347-377.

28. Shenderova OA, Zhirnov VV, Brenner DW. Carbon nanostructures. Critical Reviews in Solid State and Materials Sciences. 2002;27(3-4): 227-356.

29. Baidakova M, Vul A. New prospects and frontiers of nanodiamond clusters. J Phys D Appl Phys. 2007;40(20):6300-6311. DOI:10.1088/00223727/40/20/S14

30. Aleksenskii AE, Baidakova MV, Vul' AY, Siklitskii VI. The structure of diamond nanoclusters. Phy Solid State. 1999;41(4):668-671.

31. Shenderova OA, Hens SAC. Detonation nanodiamond particles processing, modification and bioapplications. In: Ho D, editor. Nanodiamonds: Applications in Biology and Nanoscale Medicine. New York, NY: Springer; 2010:79-116.

32. Pichot V, Comet M, Fousson E, et al. An efficient purification method for detonation nanodiamonds. Diam Relat Mater. 2008;17(1):13-22.

33. Osswald S, Yushin G, Mochalin V, Kucheyev SO, Gogotsi Y. Control of $\mathrm{sp} 2 / \mathrm{sp} 3$ carbon ratio and surface chemistry of nanodiamond powders by selective oxidation in air. J Am Chem Soc. 2006;128(35): 11635-11642.

34. Petrov I, Shenderova O, Grishko V, et al. Detonation nanodiamonds simultaneously purified and modified by gas treatment. Diam Relat Mater. 2007;16(12):2098-2103

35. Krüger A, Kataoka F, Ozawa M, et al. Unusually tight aggregation in detonation nanodiamond: identification and disintegration. Carbon. 2005;43(8):1722-1730.

36. Krueger A. The structure and reactivity of nanoscale diamond. JMater Chem. 2008;18(13):1485-1492.

37. Jiang T, Xu K. FTIR study of ultradispersed diamond powder synthesized by explosive detonation. Carbon. 1995;33(12):1663-1671.

38. Yur'ev GS, Dolmatov VY. X-ray diffraction study of detonation nanodiamonds. Journal of Superhard Materials. 2010;32(5):311-328.

39. Hawelek L, Brodka A, Dore JC, Honkimaki V, Tomita S, Burian A. Structural studies of nanodiamond by high-energy X-ray diffraction. Diam Relat Mater. 2008;17(7-10):1186-1193.

40. Dolmatov VY. On elemental composition and crystal-chemical parameters of detonation nanodiamonds. Journal of Superhard Materials. 2009;31(3):158-164.

41. Mykhaylyk OO, Solonin YM, Batchelder DN, Brydson R. Transformation of nanodiamond into carbon onions: A comparative study by high-resolution transmission electron microscopy, electron energy-loss spectroscopy, x-ray diffraction, small-angle x-ray scattering, and ultraviolet Raman spectroscopy. J Appl Phys. 2005; 97(7):074302-1-074302-16.

42. Dolmatov VY. Detonation nanodiamonds in oils and lubricants. Journal of Superhard Materials. 2010;32(1):14-20.

43. Schrand AM, Hens SA, Shenderova OA. Nanodiamond particles: properties and perspectives for bioapplications. Critical Reviews in Solid State and Materials Sciences. 2009;34(1-2):18-74.

44. Bondar VS, Pozdnyakova IO, Puzyr AP. Applications of nanodiamonds for separation and purification of proteins. Physics of the Solid State. 2004;46(4):758-760.

45. Purtov KV, Puzyr AP, Bondar VS. Nanodiamond sorbents: new carriers for column chromatography of proteins. Dokl Biochem Biophys. 2008;419:72-74.
46. Dolmatov VY. Applications of detonation nanodiamond. In: Shenderova OA, Gruen DM, editors. Ultrananocrystalline Diamond: Synthesis, Properties, and Applications. New York, NY: William Andrew; 2006:477-527.

47. Pentecost A, Gour S, Mochalin V, Knoke I, Gogotsi Y. Deaggregation of nanodiamond powders using salt- and sugar-assisted milling. ACS Appl Mater Interfaces. 2010;2(11):3289-3294.

48. Krüger A, Liang Y, Jarre G, Stegk J. Surface functionalisation of detonation diamond suitable for biological applications. J Mater Chem. 2006;16(24):2322-2328.

49. Barnard AS. Self-assembly in nanodiamond agglutinates. JMater Chem. 2008;18(34):4038-4041.

50. Chang LY, Ōsawa E, Barnard AS. Confirmation of the electrostatic self-assembly of nanodiamonds. Nanoscale. 2011;3(3):958-962.

51. Ozawa M, Inaguma M, Takahashi M, Kataoka F, Krüger A, Ōsawa E. Preparation and behavior of brownish, clear nanodiamond colloids. $A d v$ Mater. 2007;19(9):1201-1206.

52. Liang Y, Ozawa M, Krueger A. A general procedure to functionalize agglomerating nanoparticles demonstrated on nanodiamond. ACS Nano. 2009;3(8):2288-2296.

53. Ōsawa E. Recent progress and perspectives in single-digit nanodiamond. Diam Relat Mater. 2007;16(12):2018-2022.

54. Eidelman ED, Siklitsky VI, Sharonova LV, et al. A stable suspension of single ultrananocrystalline diamond particles. Diam Relat Mater. 2005;14(11-12):1765-1769.

55. Suslick KS, Hammerton DA, Cline RE. The sonochemical hot spot. J Am Chem Soc. 1986;108(18):5641-5642.

56. Aleksenskii AE, Baidakova MV, Vul' AY, Davydov VY, Pevtsova YA. Diamond-graphite phase transition in ultradisperse-diamond clusters. Physics of the Solid State. 1997;39(6):1007-1015.

57. Xu X, Yu Z, Zhu Y, Wang B. Effect of sodium oleate adsorption on the colloidal stability and zeta potential of detonation synthesized diamond particles in aqueous solutions. Diam Relat Mater. 2005;14(2): 206-212.

58. Morita Y, Takimoto T, Yamanaka H, et al. A facile and scalable process for size-controllable separation of nanodiamond particles as small as 4 nm. Small. 2008;4(12):2154-2157.

59. Tsubota T, Tanii S, Ida S, Nagata M, Matsumoto Y. Chemical modification of diamond surface with various carboxylic acids by radical reaction in liquid phase. Diam Relat Mater. 2004;13(4-8):1093-1097.

60. Ida S, Tsubota T, Tanii S, Nagata M, Matsumoto Y. Chemical modification of the diamond surface using benzoyl peroxide and dicarboxylic acids. Langmuir. 2003;19(23):9693-9698

61. Ando T, Nishitani-Gamo M, Rawles RE, Yamamoto K, Kamo M, Sato Y. Chemical modification of diamond surfaces using a chlorinated surface as an intermediate state. Diam Relat Mater. 1996;5(10): 1136-1142.

62. Liu Y, Gu Z, Margrave JL, Khabashesku VN. Functionalization of nanoscale diamond powder: fluoro-, alkyl-, amino-, and amino acidnanodiamond derivatives. Chem Mater. 2004;16(20):3924-3930.

63. Krueger A, Boedeker T. Deagglomeration and functionalisation of detonation nanodiamond with long alkyl chains. Diam Relat Mater. 2008;17(7-10):1367-1370.

64. Krueger A, Ozawa M, Jarre G, Liang Y, Stegk J, Lu L. Deagglomeration and functionalisation of detonation diamond. Physica Status Solidi (A): Applications and Materials. 2007;204(9):2881-2887.

65. Krueger A, Stegk J, Liang Y, Lu L, Jarre G. Biotinylated nanodiamond: simple and efficient functionalization of detonation diamond. Langmuir. 2008;24(8):4200-4204.

66. Kaur R, Chitanda JM, Michel D, et al. Lysine-functionalized nanodiamonds: synthesis, physiochemical characterization, and nucleic acid binding studies. Int J Nanomedicine. 2012;7:3851-3866.

67. Schrand AM, Huang H, Carlson C, et al. Are diamond nanoparticles cytotoxic? J Phys Chem B. 2007;111(1):2-7.

68. Schrand AM, Dai L, Schlager JJ, Hussain SM, Osawa E. Differential biocompatibility of carbon nanotubes and nanodiamonds. Diam Relat Mater. 2007;16(12):2118-2123. 
69. Yu SJ, Kang MW, Chang HC, Chen KM, Yu YC. Bright fluorescent nanodiamonds: no photobleaching and low cytotoxicity. $\mathrm{J}$ Am Chem Soc. 2005;127(50):17604-17605.

70. Liu KK, Cheng CL, Chang CC, Chao JI. Biocompatible and detectable carboxylated nanodiamond on human cell. Nanotechnology. 2007; 18(32):325102.

71. Chao JI, Perevedentseva E, Chung PH, et al. Nanometer-sized diamond particle as a probe for biolabeling. Biophys J. 2007;93(6): 2199-2208.

72. Huang H, Pierstorff E, Osawa E, Ho D. Active nanodiamond hydrogels for chemotherapeutic delivery. Nano Lett. 2007;7(11):3305-3314.

73. Li J, Zhu Y, Li W, Zhang X, Peng Y, Huang Q. Nanodiamonds as intracellular transporters of chemotherapeutic drug. Biomaterials. 2010; 31(32):8410-8418.

74. Xing Y, Xiong W, Zhu L, Osawa E, Hussin S, Dai L. DNA damage in embryonic stem cells caused by nanodiamonds. ACS Nano. 2011;5(3): 2376-2384.

75. Marcon L, Riquet F, Vicogne D, Szunerits S, Bodart JF, Boukherroub R. Cellular and in vivo toxicity of functionalized nanodiamond in Xenopus embryos. J Mater Chem. 2010;20(37):8064-8069.

76. Liu KK, Wang CC, Cheng CL, Chao JI. Endocytic carboxylated nanodiamond for the labeling and tracking of cell division and differentiation in cancer and stem cells. Biomaterials. 2009;30(26): 4249-4259.

77. Zhu L, Chang DW, Dai L, Hong Y. DNA damage induced by multiwalled carbon nanotubes in mouse embryonic stem cells. Nano Lett. 2007;7(12):3592-3597.

78. Zhu Y, Li W, Li Q, et al. Effects of serum proteins on intracellular uptake and cytotoxicity of carbon nanoparticles. Carbon. 2009;47(5): 1351-1358.

79. Drescher D, Orts-Gil G, Laube G, et al. Toxicity of amorphous silica nanoparticles on eukaryotic cell model is determined by particle agglomeration and serum protein adsorption effects. Anal Bioanal Chem. 2011;400(5):1367-1373.

80. Kittler S, Greulich C, Gebauer JS, et al. The influence of proteins on the dispersability and cell-biological activity of silver nanoparticles. J Mater Chem. 2010;20(3):512-518.

81. Casey A, Davoren M, Herzog E, Lyng FM, Byrne HJ, Chambers G. Probing the interaction of single walled carbon nanotubes within cell culture medium as a precursor to toxicity testing. Carbon. 2007;45(1): 34-40.

82. Guo L, Von Dem Bussche A, Buechner M, Yan A, Kane AB, Hurt RH. Adsorption of essential micronutrients by carbon nanotubes and the implications for nanotoxicity testing. Small. 2008;4(6):721-727.

83. Puzyr AP, Baron AV, Purtov KV, et al. Nanodiamonds with novel properties: a biological study. Diam Relat Mater. 2007;16(12): 2124-2128.

84. Yuan Y, Wang X, Jia G, et al. Pulmonary toxicity and translocation of nanodiamonds in mice. Diam Relat Mater. 2010;19(4):291-299.

85. Chow EK, Zhang XQ, Chen M, et al. Nanodiamond therapeutic delivery agents mediate enhanced chemoresistant tumor treatment. Sci Transl Med. 2011;3(73):73ra21.

86. Faklaris O, Garrot D, Joshi V, et al. Detection of single photoluminescent diamond nanoparticles in cells and study of the internalization pathway. Small. 2008;4(12):2236-2239.

87. Wee TL, Mau YW, Fang CY, Hsu HL, Han CC, Chang HC. Preparation and characterization of green fluorescent nanodiamonds for biological applications. Diam Relat Mater. 2009;18(2-3):567-573.

88. Faklaris O, Joshi V, Irinopoulou T, et al. Photoluminescent diamond nanoparticles for cell labeling: study of the uptake mechanism in mammalian cells. ACS Nano. 2009;3(12):3955-3962.

89. Martín R, Álvaro M, Herance JR, García H. Fenton-treated functionalized diamond nanoparticles as gene delivery system. ACS Nano. 2010;4(1):65-74.

90. Li X, Shao J, Qin Y, Shao C, Zheng T, Ye L. TAT-conjugated nanodiamond for the enhanced delivery of doxorubicin. J Mater Chem. 2011;21(22):7966-7973.
91. Liu KK, Zheng WW, Wang CC, et al. Covalent linkage of nanodiamond-paclitaxel for drug delivery and cancer therapy. Nanotechnology. 2010;21(31):315106.

92. Chen M, Pierstorff ED, Lam R, et al. Nanodiamond-mediated delivery of water-insoluble therapeutics. ACS Nano. 2009;3(7):2016-2022.

93. Mohanraj VJ, Chen Y. Nanoparticles - a review. Trop J Pharm Res. 2006;5(1):561-573.

94. Sinha R, Kim GJ, Nie S, Shin DM. Nanotechnology in cancer therapeutics: bioconjugated nanoparticles for drug delivery. $\mathrm{Mol}$ Cancer Ther. 2006;5(8):1909-1917.

95. Goga A, Yang D, Tward AD, Morgan DO, Bishop JM. Inhibition of CDK1 as a potential therapy for tumors over-expressing MYC. Nat Med. 2007;13(7):820-827.

96. Nayfield SG, Karp JE, Ford LG, Dorr FA, Kramer BS. Potential role of tamoxifen in prevention of breast cancer. $J$ Natl Cancer Inst. 1991;83(20):1450-1459.

97. Fisher B, Costantino JP, Wickerham DL, et al. Tamoxifen for the prevention of breast cancer: current status of the National Surgical Adjuvant Breast and Bowel Project P-1study. J Natl Cancer Inst. 2005;97(22):1652-1662.

98. Liang XJ, Chen C, Zhao Y, Wang PC. Circumventing tumor resistance to chemotherapy by nanotechnology. Methods Mol Biol. 2010;596: 467-488.

99. Peer D, Karp JM, Hong S, Farokhzad OC, Margalit R, Langer R. Nanocarriers as an emerging platform for cancer therapy. Nat Nanotechnol. 2007;2(12):751-760.

100. Chidambaram M, Manavalan R, Kathiresan K. Nanotherapeutics to overcome conventional cancer chemotherapy limitations. $J$ Pharm Pharm Sci. 2011;14(1):67-77.

101. Shimkunas RA, Robinson E, Lam R, et al. Nanodiamond-insulin complexes as $\mathrm{pH}$-dependent protein delivery vehicles. Biomaterials. 2009;30(29):5720-5728.

102. Liu Y, Petreaca M, Yao M, Martins-Green M. Cell and molecular mechanisms of keratinocyte function stimulated by insulin during wound healing. BMC Cell Biol. 2009;10:1.

103. Schneider LA, Korber A, Grabbe S, Dissemond J. Influence of $\mathrm{pH}$ on wound-healing: a new perspective for wound-therapy? Arch Dermatol Res. 2007;298(9):413-420.

104. Wiethoff CM, Middaugh CR. Barriers to nonviral gene delivery. J Pharm Sci. 2003;92(2):203-217.

105. Gary DJ, Puri N, Won YY. Polymer-based siRNA delivery: perspectives on the fundamental and phenomenological distinctions from polymerbased DNA delivery. J Control Release. 2007;121(1-2):64-73.

106. Whitehead KA, Langer R, Anderson DG. Knocking down barriers: advances in siRNA delivery. Nat Rev Drug Discov. 2009;8(2): 129-138.

107. Takahashi Y, Nishikawa M, Takakura Y. Nonviral vector-mediated RNA interference: its gene silencing characteristics and important factors to achieve RNAi-based gene therapy. Adv Drug Deliv Rev. 2009;61(9):760-766.

108. van de Water FM, Boerman OC, Wouterse AC, Peters JG, Russel FG, Masereeuw R. Intravenously administered short interfering RNA accumulates in the kidney and selectively suppresses gene function in renal proximal tubules. Drug Metab Dispos. 2006;34(8):1393-1397.

109. Zhang XQ, Chen M, Lam R, Xu X, Osawa E, Ho D. Polymerfunctionalized nanodiamond platforms as vehicles for gene delivery. ACS Nano. 2009;3(9):2609-2616.

110. Chen M, Zhang XQ, Man HB, Lam R, Chow EK, Ho D. Nanodiamond vectors functionalized with polyethylenimine for siRNA delivery. J Phys Chem Lett. 2010;1(21):3167-3171.

111. Ramdas AK. Electronic excitations in isotopically controlled diamonds: infrared and Raman spectroscopy of acceptor-bound holes. In: Nazaré MH, Neves AJ, editors. Properties, Growth and Applications of Diamond. London: INSPEC; 2001:21-27.

112. Kiflawi I, Lawson SC. Aggregates of nitrogen in diamond. In: Nazare MH, Neves AJ, editors. Properties, Growth and Applications of Diamond. London: INSPEC; 2001:130-133. 
113. Vaijayanthimala V, Chang HC. Functionalized fluorescent nanodiamonds for biomedical applications. Nanomedicine (Lond). 2009:4(1):47-55.

114. Mainwood A. Nitrogen and nitrogen-vacancy complexes and their formation in diamond. Phys Rev B Condes Matter. 1994;49(12): 7934-7940.

115. Neugart F, Zappe A, Jelezko F, et al. Dynamics of diamond nanoparticles in solution and cells. Nano Lett. 2007;7(12):3588-3591.

116. Treussart F, Jacques V, Wu E, Gacoin T, Grangier P, Roch JF. Photoluminescence of single colour defects in $50 \mathrm{~nm}$ diamond nanocrystals. Physica B Condens Matter. 2006;376-377:926-929.

117. Davies G, Hamer MF. Optical studies of the $1.945 \mathrm{eV}$ vibronic band in diamond. Proc R Soc Lond A Mat Phys Sci. 1976;348(1653): 285-298.

118. Wee TL, Tzeng YK, Han CC, et al. Two-photon excited fluorescence of nitrogen-vacancy centers in proton-irradiated type $\mathrm{Ib}$ diamond. J Phys Chem A. 2007;111(38):9379-9386.

119. Lawson SC, Fisher D, Hunt DC, Newton ME. On the existence of positively charged single-substitutional nitrogen in diamond. $J$ Phys Condens Matter. 1998;10(27):6171-6180.

120. Hui YY, Chang YR, Mohan N, Lim TS, Chen YY, Chang HC. Polarization modulation spectroscopy of single fluorescent nanodiamonds with multiple nitrogen vacancy centers. $J$ Phys Chem $A$. 2011;115(10):1878-1884.

121. Gruber A, Dräbenstedt A, Tietz C, Fleury L, Wrachtrup J, von Borczyskowski C. Scanning confocal optical microscopy and magnetic resonance on single defect centers. Science. 1997;276(5321): 2012-2014.

122. Collins AT, Thomaz MF, Jorge MI. Luminescence decay time of the $1.945 \mathrm{eV}$ centre in type Ib diamond. Journal of Physics C: Solid State Physics. 1983;16(11):2177-2181.

123. Tisler J, Balasubramanian G, Naydenov B, et al. Fluorescence and spin properties of defects in single digit nanodiamonds. ACS Nano. 2009;3(7):1959-1965.

124. Hui YY, Cheng CL, Chang HC. Nanodiamonds for optical bioimaging. J Phys D Appl Phys. 2010;43(37):374021.

125. Gali A, Fyta M, Kaxiras E. Ab initio supercell calculations on nitrogenvacancy center in diamond: its electronic structure and hyperfine tensors. Phys Rev B. 2008;77(15):155206.

126. Weng MF, Chiang SY, Wang NS, Niu H. Fluorescent nanodiamonds for specifically targeted bioimaging: application to the interaction of transferrin with transferrin receptor. Diam Relat Mater. 2009;18(2-3): 587-591. DOI:10.1016/j.diamond.2008.07.012.

127. Koenig K, Schneckenburger H. Laser-induced autofluorescence for medical diagnosis. J Fluoresc. 1994;4(1):17-40.

128. Aubin JE. Autofluorescence of viable cultured mammalian cells. J Histochem Cytochem. 1979;27(1):36-43.

129. Lichtman JW, Conchello JA. Fluorescence microscopy. Nat Methods. 2005;2(12):910-919.

130. Resch-Genger U, Grabolle M, Cavaliere-Jaricot S, Nitschke R, Nann T. Quantum dots versus organic dyes as fluorescent labels. Nat Methods. 2008;5(9):763-775.

131. Dahan M, Laurence T, Pinaud F, et al. Time-gated biological imaging by use of colloidal quantum dots. Opt Lett. 2001;26(11): $825-827$.

International Journal of Nanomedicine

\section{Publish your work in this journal}

The International Journal of Nanomedicine is an international, peerreviewed journal focusing on the application of nanotechnology in diagnostics, therapeutics, and drug delivery systems throughout the biomedical field. This journal is indexed on PubMed Central, MedLine, CAS, SciSearch ${ }^{\circledR}$, Current Contents ${ }^{\circledR} /$ Clinical Medicine,
132. Davies G. The effect of nitrogen impurity on the annealing of radiation damage in diamond. $J$ Phys C Solid State Phys. 1972;5(17): 2534-2542.

133. Vlasov II, Shenderova O, Turner S, et al. Nitrogen and luminescent nitrogen-vacancy defects in detonation nanodiamond. Small. 2010; 6(5):687-694.

134. Borjanovic V, Lawrence WG, Hens S, et al. Effect of proton irradiation on photoluminescent properties of PDMS-nanodiamond composites. Nanotechnology. 2008;19(45):455701.

135. Kvit AV, Zhirnov VV, Tyler T, Hren JJ. Aging effect and nitrogen distribution in diamond nanoparticles. Composites Part B Eng. 2004; 35(2):163-166.

136. Vlasov II, Barnard AS, Ralchenko VG, et al. Nanodiamond photoemitters based on strong narrow-band luminescence from siliconvacancy defects. Adv Mater. 2009;21(7):808-812.

137. Barnard AS, Sternberg M. Can we predict the location of impurities in diamond nanoparticles? Diam Relat Mater. 2007;16(12): 2078-2082.

138. Smith BR, Inglis DW, Sandnes B, et al. Five-nanometer diamond with luminescent nitrogen-vacancy defect centers. Small. 2009;5(14): 1649-1653.

139. Bradac C, Gaebel T, Naidoo N, et al. Observation and control of blinking nitrogen-vacancy centres in discrete nanodiamonds. Nat Nanotechnol. 2010;5(5):345-349.

140. Turner S, Lebedev OI, Shenderova O, Vlasov II, Verbeeck J, Van Tendeloo G. Determination of size, morphology, and nitrogen impurity location in treated detonation nanodiamond by transmission electron microscopy. Adv Funct Mater. 2009;19(13):2116-2124.

141. Mkandawire M, Pohl A, Gubarevich T, et al. Selective targeting of green fluorescent nanodiamond conjugates to mitochondria in HeLa cells. J Biophotonics. 2009;2(10):596-606.

142. Mohan N, Chen CS, Hsieh HH, Wu YC, Chang HC. In vivo imaging and toxicity assessments of fluorescent nanodiamonds in Caenorhabditis elegans. Nano Lett. 2010;10(9):3692-3699. DOI:10.1021/n11021909.

143. Smith BR, Niebert M, Plakhotnik T, Zvyagin AV. Transfection and imaging of diamond nanocrystals as scattering optical labels. J Lumin. 2007;127(1):260-263.

144. Colpin Y, Swan A, Zvyagin AV, Plakhotnik T. Imaging and sizing of diamond nanoparticles. Opt Lett. 2006;31(5):625-627.

145. Perevedentseva E, Cheng CY, Chung PH, Tu JS, Hsieh YH, Cheng CL. The interaction of the protein lysozyme with bacteria $E$. coli observed using nanodiamond labelling. Nanotechnology. 2007;18(31): 315102 . DOI:10.1088/0957-4484/18/31/315102.

146. Cheng CY, Perevedentseva E, Tu JS, et al. Direct and in vitro observation of growth hormone receptor molecules in A549 human lung epithelial cells by nanodiamond labeling. Appl Phys Lett. 2007;90(16):163903.

147. Knight DS, White WB. Characterization of diamond films by Raman spectroscopy. J Mater Res. 1989;4(2):385-393.

Journal Citation Reports/Science Edition, EMBase, Scopus and the Elsevier Bibliographic databases. The manuscript management system is completely online and includes a very quick and fair peer-review system, which is all easy to use. Visit http://www.dovepress.com/ testimonials.php to read real quotes from published authors. 